\title{
Functional Changes in the Cortical Semantic Network in Amnestic Mild Cognitive Impairment
}

Jessica Pineault ${ }^{1,2}$, Pierre Jolicoeur ${ }^{1,2}$, Stephan Grimault ${ }^{3,4}$, Patrick Bermudez ${ }^{5}$, Simona Maria Brambati $^{1,2}$, Jacinthe Lacombe ${ }^{1,2}$, Juan Manuel Villalpando ${ }^{2,6,7}$, Marie-Jeanne Kergoat ${ }^{2,6,7}$ et Sven Joubert ${ }^{1,2}$

${ }^{1}$ Département de psychologie (Centre de Recherche en Neuropsychologie et Cognition), Université de Montréal, Montréal, Canada.

${ }^{2}$ Centre de Recherche, Institut Universitaire de Gériatrie de Montréal, Université de Montréal, Montréal, Canada. ${ }^{3}$ Centre National de la Recherche Scientifique, CNRS, Marseille, France.

${ }^{4}$ PERFORM Centre, Université Concordia, Montréal, Canada. ${ }^{5}$ Montreal Neurological Institute, Montreal, Canada.

${ }^{6}$ Clinique de cognition, Institut Universitaire de Gériatrie de Montréal, Université de Montréal, Montréal, Canada.

${ }^{7}$ Faculté de médecine, Université de Montréal, Montréal, Canada. 


\begin{abstract}
Semantic memory impairment has been documented in older individuals with amnestic Mild cognitive impairment (aMCI), who are at risk of developing Alzheimer's disease (AD), yet little is known about the neural basis of this breakdown. The main objective of this study was to investigate the brain mechanisms associated with semantic performance in patients with aMCI. Method: A group of aMCI patients and a group of healthy older controls carried out a semantic categorization task while their brain activity was recorded using magnetoencephalography (MEG). During the task, participants were shown famous faces and had to determine whether each famous person matched a given occupation. The main hypotheses were that: (i) semantic processing should be compromised for aMCI patients, and (ii) these deficits should be associated with cortical dysfunctions within specific areas of the semantic network. Results: Behavioural results showed that aMCI participants were significantly slower and less accurate than control participants at the semantic task, corroborating previous reports. Additionally, relative to controls, a significant pattern of hyperactivation was found in the aMCI group within specific regions of the semantic network, including the right anterior temporal lobe and inferior prefrontal cortex. Conclusions: Abnormal functional activation within key areas of the semantic network suggests that it is compromised early in the disease process. Moreover, this pattern of increased activation in aMCI was positively associated with grey matter integrity in specific areas, but was not associated with any specific pattern of atrophy, suggesting that functional hyperactivation may precede atrophy of the semantic network in aMCI.
\end{abstract}

Keywords: semantic memory (SM); famous faces; Mild cognitive impairment (MCI); magnetoencephalography (MEG); cortical thickness (CT). 


\section{Introduction}

Semantic memory (SM) encompasses general world knowledge acquired over a lifetime and shared by members of a same cultural group. Knowledge about objects, people and places, as well as knowledge about facts, concepts and language all represent different aspects of SM (Levy, Bayley, \& Squire, 2004; Tulving, 1972). While memory systems such as working memory and episodic memory decline over the course of aging, SM remains stable ; in fact the store of conceptual knowledge even improves throughout the healthy aging process (Park et al., 2002), reflecting accumulation of knowledge. In contrast, semantic impairment represents one of the earliest features of cognitive decline in neurodegenerative diseases such as Alzheimer's disease (AD) (Adlam, Bozeat, Arnold, Watson, \& Hodges, 2006; Amieva et al., 2008; Duong, Whitehead, Hanratty, \& Chertkow, 2006; Joubert et al., 2010; Joubert et al., 2008; Libon et al., 2013; Wilson, Leurgans, Boyle, \& Bennett, 2011). Studies on amnestic Mild cognitive impairment (aMCI), a condition considered by many authors to reflect a transitional stage between healthy aging and $\mathrm{AD}$, have shown that although episodic memory deficits characterize this condition, aMCI patients also have deficits in SM (Adlam et al., 2006; Ahmed, Arnold, Thompson, Graham, \& Hodges, 2008; Ally, 2012; Balthazar et al., 2011; Barbeau et al., 2012; Brambati, Peters, Belleville, \& Joubert, 2012; Carter et al., 2012; Dudas, Clague, Thompson, Graham, \& Hodges, 2005; EstevezGonzalez et al., 2004; Joubert et al., 2010; Kirchberg et al., 2012; Van Der Meulen et al., 2012; Verma \& Howard, 2012; Vogel, Gade, Stokholm, \& Waldemar, 2005). Among the various types of knowledge investigated, knowledge of famous persons appears particularly prone to conceptual breakdown in this condition (Estevez-Gonzalez et al., 2004; Joubert et al., 2010; Joubert et al., 2008; Vogel et al., 2005). For instance, aMCI individuals were found to be significantly more impaired at naming photographs of famous persons and accessing underlying biographical knowledge than common objects (Clague, Graham, Thompson, \& Hodges, 2011; Joubert et al., 2010; Joubert et al., 2008). It has been suggested that unique conceptual entities (e.g. famous persons) might be more sensitive to breakdown in AD than concepts with more generic attributes (i.e. common objects or animals) (Ahmed et al., 2008; Joubert et al., 2010; Joubert et al., 2008; Vogel et al., 2005). It has also been reported that aMCI individuals whose semantic knowledge of famous persons was most impaired had a higher rate of conversion to AD relative to nonconverters (Estevez-Gonzalez et al., 2004; Thompson, Graham, Patterson, Sahakian, \& Hodges, 
2002). Therefore, clinical and experimental measures assessing famous person knowledge appear to be particularly useful in the assessment of semantic breakdown in aMCI and may even help in predicting future conversion to AD according to certain authors (Vogel et al., 2005). The exact nature of these semantic deficits in aMCI has been subject to discussion; for instance, it remains debated whether conceptual breakdown in aMCI reflects strictly executive difficulties in the manipulation of knowledge or whether it also reflects a more central degradation of this knowledge. In a semantic priming paradigm, aMCI patients were found to show an absence of semantic priming effect relative to healthy older people (Brambati et al., 2012), pointing to a central semantic disturbance.

Although a growing number of studies have explored the nature of semantic impairment in aMCI using a variety of clinical and experimental tasks, very few have yet examined the neural basis of this impairment. One Voxel-based morphometry (VBM) study in aMCI and AD patients established a correlation between semantic performance and grey matter volume in the left anterior temporal lobe (ATL) and in the left inferior prefrontal cortex (IPC) (Joubert et al., 2010), regions respectively presumed to be involved in processing conceptual representations at an abstract and amodal level, and in the controlled processes necessary to manipulate this semantic knowledge (Noonan, Jefferies, Visser, \& Ralph, 2013; Patterson, Nestor, \& Rogers, 2007; Ralph, Jefferies, Patterson, \& Rogers, 2017; Whitney, Kirk, O'Sullivan, Ralph, \& Jefferies, 2011). Barbeau et al. (2012) also found that semantic deficits in aMCI patients correlated with significant volume reduction within several temporal lobe regions including anterior subhippocampal structures (encroaching the medial portion of the ATL) as well as the enthorinal and perirhinal cortices.

In terms of functional neuroimaging studies, only a few have investigated brain activation associated with semantic processing in aMCI patients. Using an fMRI picture naming protocol probing object knowledge, Gigi, Babai, Penker, Hendler, and Korczyn (2010) demonstrated that although aMCI patients' performance wasn't significantly impaired when compared to controls, they exhibited increased activation of prefrontal regions during the naming task. This pattern of hyperactivation was interpreted as a compensatory brain mechanism allowing patients to perform well despite structural deficits found in the parietal region and in the fusiform gyri (Gigi et al., 2010). Similarly, Woodard et al. (2009) used a low effort, high accuracy SM task to investigate recognition of famous people in aMCI patients. Results revealed that, despite levels of 
performance comparable to controls, aMCI patients exhibited increased activity in posterior parietal and temporal regions as they completed the name-discrimination task. The authors defined this pattern of increased brain activation as a reflection of compensatory recruitment of neural resources in brain regions underpinning SM systems in the context of cumulative neuropathology (Woodard et al., 2009). Similar patterns of functional hyperactivation have also been found in aMCI patients relative to healthy older controls in the medial temporal lobe region, namely the hippocampus, using a variety of episodic memory paradigms (i.e. face-name, object, and word encoding paradigms) (Dickerson et al., 2005; Dickerson \& Sperling, 2008; Hämäläinen, Tervo, et al., 2007; Kircher et al., 2007). These patterns of functional hyperactivation, which were absent in $\mathrm{AD}$ patients, were also interpreted as reflecting specific mechanisms necessary to compensate for the progression of the disease in the brain at this particular stage (Dickerson et al., 2005).

The aim of the current study was to conduct an in vivo investigation of the brain mechanisms associated with semantic processing of famous persons in aMCI and healthy older individuals using magnetoencephalography (MEG). MEG is a non-invasive imaging technique used to record the magnetic fields generated by cortical electric activity. In contrast to other neuroimaging techniques such as fMRI, MEG has an excellent temporal resolution. Another crucial advantage of MEG is that it allows to record brain activity while obtaining a good signal in the ATL (i.e. the temporal poles), a region that is susceptible to significant signal reduction and which has been found difficult to activate in classic fMRI protocols (Devlin et al., 2000; Smith, Matthews, \& Jezzard, 2001; Visser, Jefferies, \& Ralph, 2010). More specifically, a group of aMCI patients and a group of healthy older adults carried out a semantic paradigm of visually presented faces of famous persons in which they had to correctly categorize pictures of famous people based on their occupation. Prior to the presentation of each face, the written name of a category (athletes, singers, actors, or political figures) was presented on the screen and the participants had to decide if the famous face matched the occupation or not (for example, George Bush - athlete). Analyses were conducted on the signal around the N400, a well-studied electrophysiological marker of semantic processing (see Kutas and Federmeier (2011) for a review) elicited by any material requiring access to SM, namely famous/familiar faces (Barrett \& Rugg, 1989; Dietl et al., 2005; Jemel, George, Olivares, Fiori, \& Renault, 1999; Smith \& Halgren, 1987). Indeed, although classically described in the context of semantic incongruity, the N400 is now more widely 
conceptualized as a cortical response reflecting "processing of meaning" within brain regions part of a distributed semantic network following the presentation of a word or an image, regardless of modality, and thus appears to reflect a specifically semantic (amodal) process (Besson, Magne, \& Regnault, 2004; Federmeier \& Kutas, 2001; Kutas \& Federmeier, 2011).

Our main hypothesis was that MEG would reveal patterns of abnormal functional activation in aMCI associated with altered semantic processing within key regions of the semantic network, such as the ATL and the IPC regions. Patterns of abnormal activation within these regions in aMCI patients may provide supporting evidence in favour of neuroanatomical disruption underlying both "central" conceptual processing (ATL region) and semantic controlled processes (IPC and posterior temporal region). Moreover, we predicted that aMCI patients would show poorer accuracy and longer reaction times while performing the semantic task when compared with controls.

\section{Materials and Methods}

\section{Participants}

A group of aMCI patients and a group of healthy older controls matched for age and education participated in the present study. All participants gave written informed consent before participating to the study, and the study was approved by the Research Ethics Board of the Institut Universitaire de Gériatrie de Montréal (IUGM). Since semantic tests are particularly sensitive to cultural background, all participants included in the study were required to have lived in Quebec at least during the previous 40 years and spoke French as their native tongue. Among 46 aMCI patients initially referred for the current study, only 14 right-handed participants (mean age $=75.4$, standard deviation $(\mathrm{SD})=7.1 ; 10$ females and 4 males) were selected in the final aMCI group, due to the stringent requirements of MEG and MRI testing and based on exclusionary criteria (for more information, see exclusionary criteria listed in the next paragraph). MCI patients were referred by specialists at the Memory Clinic of the IUGM in Montreal, where they had been previously assessed and diagnosed by a team of expert neurologists, geriatricians and neuropsychologists. At the Clinic, a thorough case history (including clinical scales such as Hachinski's to assess vascular risk) and physiological examination were conducted to exclude any neurological or cardiovascular disease susceptible to account for the cognitive impairment. Patients showed no impairment in 
activities of daily living, as assessed during a clinical interview (Lawton \& Brody, 1969) and their MMSE (Mini-Mental State Examination) showed no global impairment using a cut-off score for age and education $(\geq 25 / 30)$ (Folstein, Folstein, \& McHugh, 1975). MCI patients corresponded to Petersen's criteria (Petersen et al., 2001). Criteria also included a memory complaint confirmed by an informant, as well as an objective memory impairment evidenced through a formal neuropsychological assessment. Objective memory impairment was defined here as performance exceeding a cut-off score of $-1.5 \mathrm{SD}$ below the mean of matched normal older participants on standard measures of episodic memory. Ultimately, aMCI patients were not sufficiently impaired both on a cognitive and functional level to meet DSM-IV criteria for AD or dementia.

The control group included 14 healthy older adults (mean age $=73.7, \mathrm{SD}=5.8 ; 10$ females and 4 males) with normal cognitive function as confirmed by their performance on a general neuropsychological assessment. Controls were recruited using the IUGM Research Centre participant database and through written public announcements. They were matched to the aMCI group for age and education level. Basic exclusionary criteria for both the aMCI and healthy control participants included a history of systemic, cerebrovascular, neurological or neurodegenerative diseases, past or current psychiatric illness, traumatic brain injury, history of alcoholism, untreated medical or metabolic condition (such as diabetes and hypothyroidism for instance), general anesthesia in the last year as well as uncorrected hearing and vision problems. Prior to testing, participants were also screened for possible MEG and MRI artifacts due to dental work or metal implants (pacemaker, clips, prostheses, insulin pumps, tattoos and permanent makeup, etc.).

A Mann-Whitney $U$ test indicated that both groups did not differ significantly in terms of age $(U=75.5, z=-1.038, p=.306)$ or education $(U=89.5, z=-.394, p=.701)$. As expected, however, the two groups differed significantly in terms of their MMSE $(U=141.5, z=-2.703$, $\mathrm{p}<.05)$ and MoCA scores $(U=153.5, z=3.596, p<.001)$.

\section{Neuropsychological Assessment}

The two groups underwent a detailed neuropsychological assessment, which included neuropsychological measures of memory, language, executive functions and visuospatial abilities. Episodic memory was assessed with the RL/RI 16 (Van Der Linden et al., 2004), which is a 
free/cued 16 word recall test generally used as a measure of verbal learning in French-speaking populations (a French adaptation of the Free and Cued Selective Reminding Test). General semantic abilities were assessed using the Pyramids and Palm Trees test (Howard \& Patterson, 1992). Visuoconstructional abilities were evaluated using the copy of the Rey-Osterrieth complex figure (Rey, Wallon, \& Mesmin, 1960), and the immediate (3 min) and delayed (20 min) recall of the figure was used to assess visual memory. The 15-item version of the Boston Naming Test was administered to participants in order to evaluate their language skills (Calero, Arnedo, Navarro, Ruiz-Pedrosa, \& Carnero, 2002), as well as the Letter (P) and Category (animals) fluency tests (Cardebat, Doyon, Puel, Goulet, \& Joanette, 1990). The Stroop Victoria test (Regard, 1981) and parts A and B of the Trail Making Test (Reitan, 1955) were used to evaluate executive functions. Short-term and working memory was measured using the forward and backward span subtests of the Weschler Memory Scale (Wechsler, 1997). The incomplete letters task of the Visual Object and Space Perception battery (VOSP) (Warrington \& James, 1991) was used to assess primary visuoperceptual processing. The Benton Facial Recognition test was used to assess face processing abilities (Benton, 1994). Finally, the Geriatric Depression Scale (Yesavage et al., 1983) was administered to participants to ensure that they did not suffer from any form of depressive state that may have had an impact on their cognitive functions (see Table 1 for results of neuropsychological assessment).

Table 1. Group demographics and neuropsychological test results.

\begin{tabular}{lccc}
\hline Variables & Controls $(\mathrm{n}=14)$ & aMCI $(\mathrm{n}=14)$ & $p$ value* \\
\hline Demographics & & & \\
Age & $73.7(5.8)$ & $75.4(7.1)$ & $\mathrm{Ns}$ \\
Education & $15.0(4.3)$ & $15.6(3.1)$ & $\mathrm{Ns}$ \\
Gender (M/F) & $4 / 10$ & $4 / 10$ & $\mathrm{Ns}$ \\
\hline Memory & & & \\
RL/RI 16 & & & \\
$\quad$ Immediate free recall of a word list (16) & $9.4(2.2)$ & $6.7(3.9)$ & $p<.05$ \\
$\quad$ Immediate total recall of a word list (16) & $15.8(0.4)$ & $11.9(3.5)$ & $p<.01$ \\
$\quad 13.7(2.0)$ & $7.3(4.4)$ & $p<.001$ \\
$\quad$ Delayed free recall of a word list (16) & $15.9(0.4)$ & $13.2(2.8)$ & $p<.01$ \\
$\quad$ Recognition in a word list (48) & $47.7(0.5)$ & $45.8(2.4)$ & $p<.05$ \\
Rey-Osterrieth Complex Figure & & & \\
$\quad$ Immediate recall (36) & $16.8(6.4)$ & $9.3(5.1)$ & $p<.005$ \\
$\quad$ Delayed recall (36) & $16.2(6.0)$ & $10.0(5.9)$ & $p<.05$ \\
\hline
\end{tabular}




\begin{tabular}{|c|c|c|c|}
\hline $\begin{array}{l}\text { Semantic knowledge } \\
\text { Pyramids and Palm Trees Test (52) }\end{array}$ & $50.9(1.1)$ & $50.2(1.6)$ & Ns \\
\hline \multicolumn{4}{|c|}{ Executive function / working memory } \\
\hline \multicolumn{4}{|c|}{ Stroop-Victoria Test (sec.) } \\
\hline Part A (dots) & $14.0(3.2)$ & $15.5(4.2)$ & Ns \\
\hline Part B (words) & $17.7(2.8)$ & $21.7(5.4)$ & $p<.05$ \\
\hline Part C (interference) & $30.8(9.1)$ & $34.5(9.1)$ & Ns \\
\hline \multicolumn{4}{|l|}{ Trail Making Test (sec.) } \\
\hline Part $A$ & $49.4(29.2)$ & $55.7(19.9)$ & Ns \\
\hline Part $B$ & $78.2(34.1)$ & $126.2(41.7)$ & $p<.005$ \\
\hline Digit span forward (WAIS-III) & $10.1(2.1)$ & $8.7(1.6)$ & Ns \\
\hline Digit span backward (WAIS-III) & $7.3(2.4)$ & $6.1(2.1)$ & Ns \\
\hline \multicolumn{4}{|l|}{ Language } \\
\hline Naming (Boston Naming Test) (15) & $14.1(2.0)$ & $13.1(1.5)$ & Ns \\
\hline Verbal fluency "P" in $2 \mathrm{~min}$ & $24.7(7.4)$ & $23.2(6.1)$ & Ns \\
\hline Category fluency "animals" in $2 \mathrm{~min}$ & $25.5(8.2)$ & $22.2(6.4)$ & Ns \\
\hline \multicolumn{4}{|l|}{ Visuoconstructional abilities } \\
\hline \multicolumn{4}{|l|}{ Rey-Osterrieth Complex Figure } \\
\hline Copy (36) & $31.6(3.8)$ & $27.6(7.3)$ & Ns \\
\hline \multicolumn{4}{|l|}{ Visuoperceptual abilities } \\
\hline Benton's Facial Recognition Test (54) & $48.0(3.2)$ & $45.0(5.1)$ & Ns \\
\hline \multicolumn{4}{|l|}{ Visual object and space perception battery } \\
\hline Incomplete letters (20) & $19.5(1.0)$ & $19.4(0.7)$ & Ns \\
\hline \multicolumn{4}{|l|}{ Affect } \\
\hline Geriatric Depression Scale (GDS) & $2.3(2.4)$ & $1.3(2.1)$ & Ns \\
\hline
\end{tabular}

\section{Semantic decision task}

This computerized task was developed in the Laboratory and specifically adapted to MEG. It was devised to evaluate the integrity of semantic processing through a semantic decision task addressing knowledge of famous persons. Stimuli consisted of 192 high resolution coloured photographs of famous faces belonging to four different occupational categories (athletes, singers, actors or political figures). Famous faces were those of individuals who had become famous at different periods of time ranging from the 1930s to the present. Stimuli were equivalent in terms of size and were ridden of any background or cues which may have provided contextual information about the person, such as the type of clothing, distinctive attributes, etc. which may have helped participants in their decisions (ref. Appendix A). Stimuli were back projected on a 
computer screen located at eye level at a distance of $60 \mathrm{~cm}$ from the subject in the MEG using the E-Prime software (Psychology Software Tools, Pittsburg, PA). Stimuli were presented one by one.

The semantic decision task was administered to the participants individually during a twohour meeting in the MEG lab located in the psychology department of Université de Montréal. Upon arrival, participants were tested for MEG compatibility before they were given the instructions relative to the semantic decision task. Once installed into the magnetically shielded room, each participant completed a session of testing consisting of 48 practice trials followed by 192 experimental trials. All trials started with concise written instructions reminding the participants to always keep their eyes on the fixation cross located at the centre of the screen and to blink only once feedback was given to them. Participants initiated trials by pressing simultaneously with their thumbs on both side buttons located on the response case. At the beginning of each of the four blocks, the written name of a semantic category (e.g., 'actor') was presented on the screen during $1000 \mathrm{~ms}$. The subject was instructed to decide if each of the 48 stimuli presented subsequently for a duration of $500 \mathrm{~ms}$ belonged to that semantic category and to provide an answer by pressing the appropriate key on the response cases. Each of the 96 famous faces ( 24 per category) was presented twice in the experiment: once in a matching condition (e.g., politician - George Bush) and once in a non-matching condition (e.g., actor - George Bush). Participants were given $2500 \mathrm{~ms}$ to provide their answer and were instructed to respond as accurately yet as quickly as possible after stimulus presentation. The order of blocks and the hand used to for each type of answer (matching, non-matching) was counterbalanced between participants, as well as the order of presentation of the stimuli in each block, which was randomized for each subject. A list of the stimuli is presented in Table 3 in Appendix B. To ensure that the participants did not develop performance anxiety and remained focused throughout the task, feedback relative to accuracy was not given. The only feedback shown to them was a blank circle post-response to inform them that their answer had been correctly recorded.

\section{Data Acquisition}

Functional brain activity of participants was recorded while they completed the semantic decision task using a whole-head CTF-VSM 275 sensors MEG system in a magnetically shielded room. MEG signals were acquired continuously during the task at a sampling rate of $1200 \mathrm{~Hz}$. 
Prior to recording, participants were screened for MEG artifacts (dental work/metal implants). When necessary, plastic lenses were installed on the participants' eyes to avoid magnetic perturbations from the metal parts of their personal glasses and ensure their optimal visual processing of the stimuli during the task. Bipolar electroocculogram (EOG) (electrodes placed at the left and right canthi for horizontal EOG and above and below the left eye for vertical EOG) as well as unipolar ECG were also recorded. Following the MEG protocol, each control and aMCI participant underwent a high resolution anatomical MRI using an optimized MPRAGE protocol with a 3.0 T Siemens TRIO MRI (Siemens, Erlanger, Germany) located at the Unité de neuroimagerie fonctionnelle in the Research Centre of the IUGM (http://www.unfmontreal.ca/siteweb/ Home_en.html). A volumetric magnetization prepared rapid gradient echo (MP-RAGE) sequence was used to acquire high-resolution T1-weighted 3D anatomical images, using the following parameters: $\mathrm{TR}=2.3 \mathrm{~s}, \mathrm{TE}=2.94 \mathrm{~ms}, \mathrm{TI}=900 \mathrm{~ms}$, flip angle $=9^{\circ}, \mathrm{FOV}=240 \times 256$, voxel size $=1 \times 1 \times 1.2 \mathrm{~mm}^{3}$. An 8 -channel head coil was used. The scans were then used to constrain source localization analyses of the MEG data. Using their respective MRI images, a 3D representation of each subject's cortex was realized through mathematical morphometry (refer to the source localization section for more details).

\section{Data Analyses}

Behavioural Data. Reaction times and number of correct responses were averaged for each participant to allow comparison between groups. Items left unanswered were recorded as incorrect responses. Mann-Whitney U tests were performed on both variables.

MEG Data. Third-order gradient noise reduction (computed with CTF software) was applied to all MEG signals before segmentation. Subsequently, signals were segmented according to the chosen time window ( -200 to $700 \mathrm{~ms}$ relative to the onset of the stimulus) and baselinecorrected on the basis of the mean activity during the $200 \mathrm{~ms}$ before the onset of the stimulus. Trials with eye movement were systematically removed following a careful inspection of the vertical and horizontal EOG traces. Trials with large movement artifacts, such as head motion exceeding $5 \mathrm{~mm}$ from baseline recording and other external noise were removed as well. Only trials with a correct response were included in the subsequent brain signal analyses. Due to artifacts in the MEG signals, three additional aMCI patients and two controls had to be excluded from the MEG 
functional analyses. Data was then averaged by categories and conditions, producing event-related fields. MEG analyses were conducted using CTF tools, BrainVisa (http://brainvisa.info) and AFNI software (Cox, 1996).

Given that the task was to assess the ability of aMCI patients to access and retrieve the semantic knowledge necessary to the proper semantic categorization of famous faces, a specific time window reflecting this type of processing was determined based on supporting evidence in the literature. A visual inspection of the maps displaying the averaged event-related magnetic field temporal curves of the control and patient groups allowed the selection of a temporal window extending from $420 \mathrm{~ms}$ to $600 \mathrm{~ms}$, This time window was determined as the possible latency of the N400 electrophysiological wavelength, which is known to reflect the processing and integration of meaningful semantic stimuli (Kutas \& Hillyard, 1980; Lau, Almeida, Hines, \& Poeppel, 2009; Lau, Phillips, \& Poeppel, 2008). These curves are shown in Figure 1. Previous electrophysiological studies that concentrated on characterizing the pattern of N400 among older individuals have demonstrated that it is generally delayed in normal aging, thus supporting the choice of a slightly delayed and extended time window range (Federmeier, Van Petten, Schwartz, \& Kutas, 2003; Harbin, Marsh, \& Harvey, 1984; Kutas \& Iragui, 1998; Taler, Klepousniotou, \& Phillips, 2009) For instance, in a study comparing individuals aged between 20 and 80 years old, the onset latency N400 effect was found to be delayed in latency by $100 \mathrm{~ms}$ in the oldest group (70s) relative to the youngest (20s) (Kutas \& Iragui, 1998). Another study contrasting a young and an older group on a semantic task found that the N400 effect in the older group was delayed in latency by $120 \mathrm{~ms}$ (Harbin et al., 1984). 


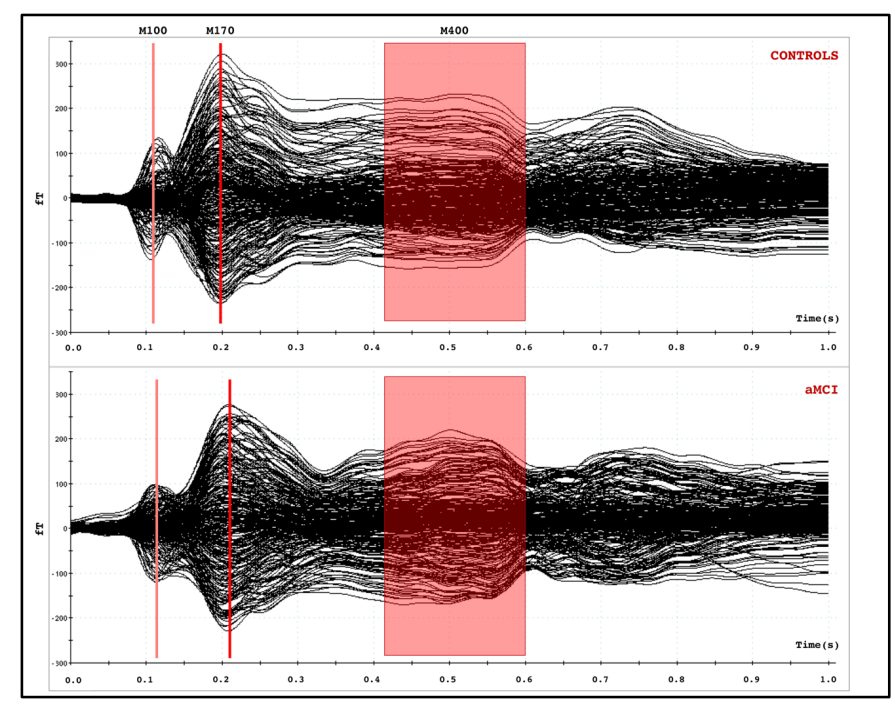

Figure 1. Averaged ERMf temporal curves of the control and patient groups

Source localization. To perform source localization, the maximum entropy on the mean method (MEM) was used (Amblard, Lapalme, \& Lina, 2004; Grova et al., 2006). This method is a cortically constrained distributed source-localization approach. The cortical surface was segmented from each anatomical MRI scan using BrainVisa software (http://brainvisa.info). Approximately 4000 sources were distributed over the entire cortical surface, and these local sources were used in distributed source localization analyses, which were conducted separately for each participant.

Following the localization analyses performed on a participant-wise basis, the following procedure was used to perform a group analysis of the localization. First, the segmented and baseline-corrected signals over trials within each condition were averaged. Then, MEM source localization was performed on the cortical surface, for each sample point of the event-related magnetic field in the analysis window (420ms to 600ms), and for each of the experimental conditions. The localization images were subsequently averaged to obtain an average cortically constrained localization map for each condition and each participant. Next, each cortical-surface average localization map was interpolated in the volume MRI image for each participant and the resulting image was smoothed using a Gaussian filter with an $8 \mathrm{~mm}$ FWHM (full width at half maximum). The source localization was constrained to the cortical surface. The smoothing produced by the Gaussian filter created a spatial extension of the sources outside to the brain. To 
remove this outside activity a mask was applied following the Gaussian filtering process. Finally, all the images were normalized to a common template in Talairach space (ICBM 152, Montreal Neurological Institute) using SPM2.

Statistical analyses. Co-variation analyses were performed using a General Linear Model (AFNI ; (Cox, 1996)) considering the normalized source-localization maps. Comparisons between groups were performed using a voxel-wise threshold at $\mathrm{p} \leq .05$, corrected for multiple comparisons with Random Field Theory (RFT) method (Worsley et al., 2002). Clusters of voxels with a volume of at least $544 \mathrm{~mm}^{3}(t=3.5)$ were considered as statistically significant based on these criteria (displayed in Figure 2a). Additional analyses examining the relationship between functional activation and accuracy on the task (using a z-score derived from the mean accuracy score of controls) were conducted on each group, using the method described above. Clusters of voxels with a volume of at least $416 \mathrm{~mm}^{3}(t=4.5)$ for the aMCI group and at least $625 \mathrm{~mm}^{3}(t=4.91)$ for the control group were considered as statistically significant based on these criteria (see Table 4 in Appendix $C$ for more details).

Cortical thickness. Cortical thickness analyses assessed differences in cortical grey-matter between groups. T1 images were registered to the ICBM152 non-linear 6th generation template with a 12-parameter linear transformation (Collins, Neelin, Peters, \& Evans, 1994; Grabner et al., 2006), RF inhomogeneity corrected (Sled, Zijdenbos, \& Evans, 1998) and tissue classified (Tohka, Zijdenbos, \& Evans, 2004; Zijdenbos, Forghani, \& Evans, 1998). Deformable models were then used to create white and grey-matter surfaces for each hemisphere separately (Kim et al., 2005; MacDonald, Kabani, Avis, \& Evans, 2000), resulting in 4 surfaces of 41962 polygons each. From these surfaces, the t-laplace metric was derived by using the Laplacian method for determining the distance between the white and grey surfaces (Haidar \& Soul, 2006; Jones, Buchbinder, \& Aharon, 2000; Lerch \& Evans, 2005). The thickness data were subsequently blurred using a $25 \mathrm{~mm}$ surface-based diffusion-blurring kernel in preparation for statistical analyses (Chung \& Taylor, 2004). Un-normalized, native-space thickness values were used in all analyses owing to the poor correlation between cortical thickness and brain volume (Ad-Dab'bagh et al., 2005; Sowell et al., 2007). Statistical thresholds at $p=.05$ were determined by the application of RFT (Chung, Worsley, Nacewicz, Dalton, \& Davidson, 2010), with the resulting lower-bounding cluster tstatistic of 3.1, as in Figure 2b. 


\section{Results}

\section{Semantic decision task}

Behavioural data collected during the semantic decision task was subjected to MannWhitney $U$ tests, which revealed significant differences between groups both in terms of mean accuracy $(U=105.5, z=1.972, p<.05, r=.4)$ and mean response time $(U=21, z=-2.9, p<$ $.01, r=.6)$. More precisely, response times were longer for aMCI patients $(M=1424 \mathrm{~ms}, S D=161)$ than healthy controls $(M=1146 \mathrm{~ms}, S D=181)$. The aMCI group was also less accurate $(M=71$ $\%, S D=7.4)$ than the control group $(M=79 \%, S D=10.2)$.

Additional analyses conducted on the accuracy scores of both groups simultaneously revealed no significant correlation between the participants' performance on the famous faces semantic task and their performance on any of the more classical neuropsychological measures of $\mathrm{SM}\left(\right.$ PPTT $r_{\mathrm{s}}=.08, \mathrm{p}=.72$; Boston Naming Task $r_{\mathrm{s}}=.27, \mathrm{p}=.20$; Category subtest of the Verbal Fluency task $\left.r_{\mathrm{s}}=.03, \mathrm{p}=.95\right)$. This remained true even when the analysis was conducted exclusively on the aMCI group (PPTT $r_{\mathrm{s}}=-.15 \mathrm{p}=.66$; Boston Naming Task $r_{\mathrm{s}}=-.2, \mathrm{p}=.56$; Category subtest of the Verbal Fluency task $r_{\mathrm{s}}=.14, \mathrm{p}=.68$ ).

\section{MEG Data}

Localization images demonstrating the differences in functional activation between aMCI patients and healthy controls are shown in Figure 2a. As indicated previously, the source localization analyses were conducted on a time window that stretched from $420 \mathrm{~ms}$ to $600 \mathrm{~ms}$ and has formerly been associated with semantic processing in an array of studies (Kutas \& Federmeier, 2011; Kutas \& Hillyard, 1980; Lau et al., 2009; Lau et al., 2008). Results of the present study demonstrate that impaired semantic processing of famous faces in aMCI is associated with a distinct pattern of functional activation in this group, in comparison with controls.

As summarized in Table 2, significant differences between groups were found within different regions of the brain, including specific regions of the semantic network. Significantly greater activation was found in several regions of the temporal lobes in the aMCI group relative to the control group. The biggest cluster of hyperactivation (9392 voxels) was found in the right ATL 
region (i.e. temporal pole BA38); stretching as far as the right fusiform gyrus (BA37/20) and encompassing regions such as the right middle/inferior temporal gyrus, the bilateral parahippocampal gyri (PHG) (BA35/36), the left angular and the posterior middle/inferior temporal region (pMTG) (BA37/39). In addition, a relatively diffuse pattern of bilateral frontal lobe hyperactivation was found in the aMCI relative to the healthy control group, in areas such as the anterior and inferior prefrontal cortex (BA45/47), the middle frontal gyrus (BA46/8/10) and the superior frontal gyrus (BA9/11). The aMCI group activated both the left (included in the main cluster) and the right lingual gyri (BA18/19) to a greater extent than the control group. Other brain regions such as the right middle occipital gyrus, and the bilateral parietal lobules showed increased activation in the aMCI group relative to healthy controls. Therefore, functional hyperactivation was found in the aMCI relative to the control group in several key regions of the semantic network, including the right ATL as well as the pMTG and IPC bilaterally (see Table 2 for details on patterns of MEG activation). In addition, a number of regions were also found to be hypoactivated in the aMCI group relative to the control group: bilaterally in the middle and inferior occipital gyri (left BA17/18, right BA19) and inferior parietal region (BA40), as well as in the left inferior temporal gyrus (BA20/21), precuneus (BA7), precentral gyrus (BA6/22), and right anterior cingulate gyrus (BA24).

Table 2. Differences of activation between aMCI and healthy controls ${ }^{1}$.

\begin{tabular}{llllllll}
\hline Region (Brodmann's Area) & x & y & z & Cluster Size & T value \\
\hline aMCI > Controls & & & & \\
\hline
\end{tabular}

\section{Frontal regions}

$\begin{array}{lccccc}\text { R. Superior Frontal Gyrus (BA11) } & 10 & 48 & -22 & 2868 & 4.39 \\ \text { R. Superior Frontal Gyrus (BA8) } & 22 & 28 & 54 & 100 & 6.07 \\ \text { R. Inferior Frontal Gyrus (BA47) } & 36 & 30 & -6 & 80 & 3.54 \\ \text { R. Superior Frontal Gyrus (BA9) } & 36 & 42 & 34 & 76 & 4.58 \\ \text { R. Middle Frontal Gyrus (BA9/46) } & 20 & 4 & 64 & 69 & 6.34\end{array}$

\footnotetext{
${ }^{1}$ Peaks of hyper and hypoactivation in the aMCI group relative to healthy controls. A minimum cluster size of 67 voxels and a significance statistical threshold at $\mathrm{p} \leq .05$ corrected for multiple comparisons have been used. Talairach (MNI) coordinates are provided.
} 
R. Inferior Frontal Gyrus (BA46/47)

L. Middle Frontal Gyrus (BA10)

L. Precentral Gyrus (BA6)

\section{Temporal regions}

R. Superior Temporal Gyrus (BA38)

R. Mid. Temp. / Fusiform Gyrus (BA21/37)

L. Angular / Mid. Temp. Gyrus (BA39)

\section{Parietal regions}

R. Superior Parietal Lobule (BA7)

R. Post. Cingulate/Precuneus (BA31/7)

L. Inferior Parietal (SM) Lobule (BA40)

\section{Occipital regions}

R. Lingual Gyrus (BA18)

R. Inferior Occipital Gyrus (BA18/19)

$\begin{array}{rrrcr}48 & 36 & -6 & 68 & 3.66 \\ -34 & 58 & 0 & 1239 & 5.15 \\ -42 & -12 & 36 & 299 & 3.85\end{array}$

$\begin{array}{lllll}36 & 16 & -38 & 9392 & 17.06\end{array}$

$\begin{array}{lllll}44 & -55 & -10 & 124 & 4.18\end{array}$

$\begin{array}{lllll}-42 & -70 & 30 & 83 & 4.76\end{array}$

$\begin{array}{rrrcr}20 & -60 & 66 & 110 & 5.98 \\ 6 & -36 & 44 & 102 & 3.63 \\ -44 & -50 & 48 & 80 & 8.79\end{array}$

$\begin{array}{lllll}10 & -54 & 4 & 871 & 3.94\end{array}$

$\begin{array}{lllll}24 & -86 & -14 & 189 & 8.48\end{array}$

\section{aMCI $<$ Controls}

\section{Frontal regions}

R. Superior Frontal Gyrus (BA6)

R. Middle Frontal Gyrus (BA9/10)

R. Precentral Gyrus

L. Precentral Gyrus (BA4/6)

L. Middle Frontal Gyrus (BA47)

$\begin{array}{rrrcc}8 & -5 & 69 & 68 & -6.35 \\ 38 & 48 & 26 & 89 & -13.52 \\ 32 & -34 & 66 & 180 & -5.65 \\ -10 & -24 & 70 & 1416 & -3.91 \\ -28 & 36 & -10 & 84 & -5.86\end{array}$

\section{Temporal regions}

L. Inferior Temporal Gyrus (BA20/21)

$\begin{array}{llll}-38 & -8 & -42 & 70\end{array}$

\section{Parietal regions}

R. Inferior Parietal Lobule (BA39/40)

R. Paracentral Lobule (BA4)

$\begin{array}{lllll}58 & -48 & 38 & 439 & -4.00\end{array}$

L. Precuneus (BA7)

$\begin{array}{lllll}8 & -37 & 64 & 178 & -3.91\end{array}$

L. Postcentral Gyrus/Inf. Par. Lobule (BA40)

$\begin{array}{lll}-22 & -81 & 46\end{array}$

155

$-3.63$

\section{Occipital regions}

R. Cuneus (BA19)

$\begin{array}{lll}-44 & -34 & 55\end{array}$

95

$-8.79$

L. Cuneus (BA17)

L. Middle Occipital Gyrus (BA18)

$\begin{array}{rrrrr}2 & -88 & 30 & 227 & -5.16 \\ -10 & -91 & 2 & 149 & -8.79 \\ -26 & -100 & 10 & 128 & -6.56\end{array}$




\section{Limbic regions}

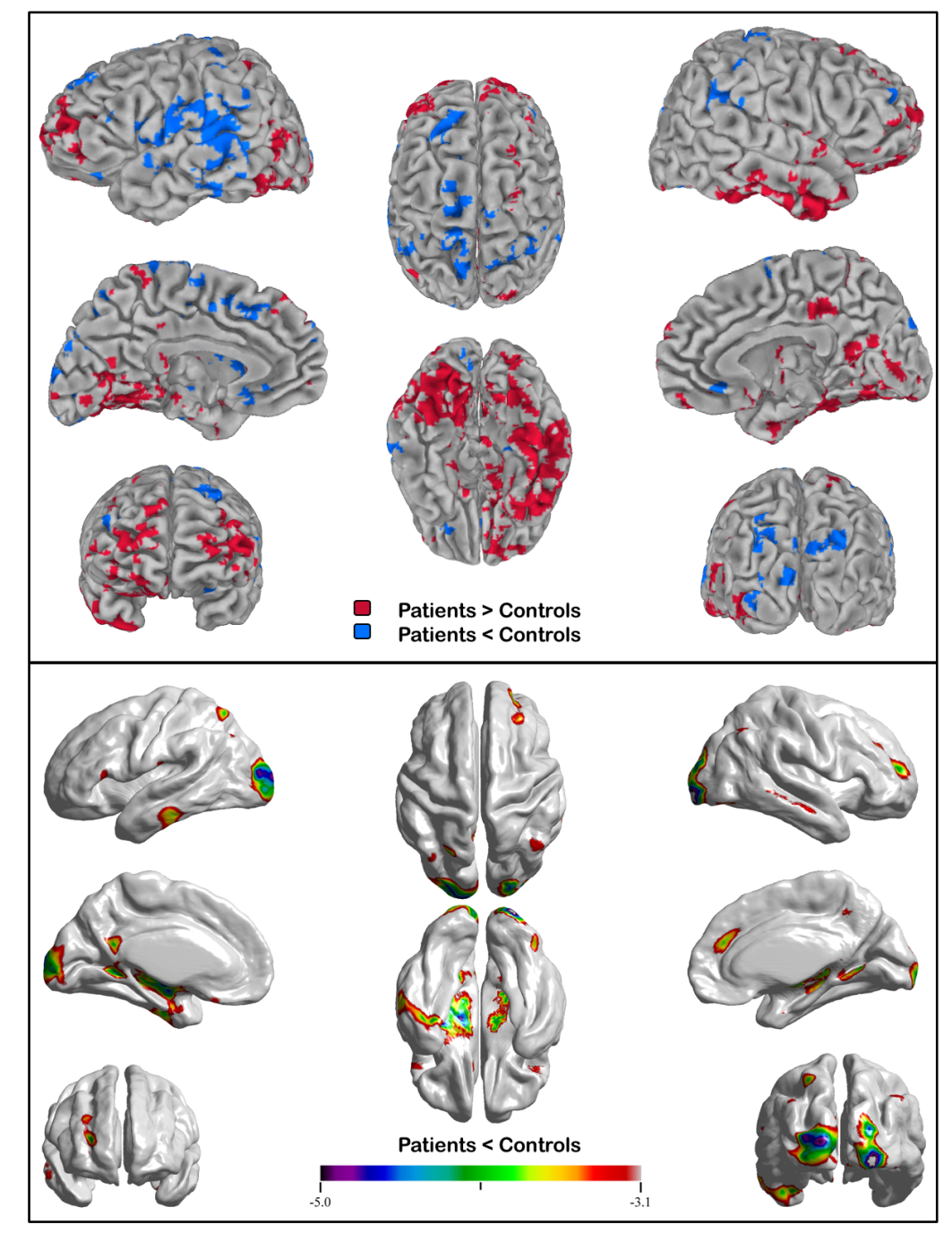

Figure 2a. Patterns of activation in the aMCI relative to the healthy control group (3D / axial and sagittal slices) / 2b. Cortical thickness anatomical differences between groups

In order to better understand how these patterns of hypo and hyperactivation were related to semantic categorization task, additional regression analyses were conducted separately within the aMCI and the control group. These analyses revealed that greater accuracy on the semantic task was associated with differential functional activation within groups. Indeed, in the aMCI 
group, better or more "preserved" performance on the semantic task (as evidenced by individual Z-scores $>-1$ SD of the healthy controls' performance) was associated with greater activation within the right ATL (BA38), inferior temporal (ITG)/fusiform region (BA20/37), right parahippocampal gyrus, and right insular region. Other regions of activation related to more accurate performance on the semantic task in the aMCI group included the left fusiform gyrus, left occipital region (BA18/19), bilateral IPC (BA45/46), and anterior cingulate gyri (BA32). In contrast, worse performance on the task in patients (individual z-scores $<-1$ SD of the healthy controls' performance) was associated with greater activation of the middle/ superior frontal region bilaterally (BA3/6/8), the left inferior/middle temporal and parahippocampal gyri, as well as the thalamus bilaterally (see Table 4 in the Appendix $\boldsymbol{C}$ for more details). Interestingly, right ATL and right ITG/fusiform task-related activation was found only in the aMCI group, while task-related prefrontal, anterior cingulate, left fusiform, occipital and parahippocampal activation appeared to be positively correlated with performance in both groups. Meanwhile, activation of the bilateral pMTG (BA21), inferior parietal regions (BA40), occipital/lingual subregions (BA18/19), and right IPC (BA45/46) was positively associated with task performance only in the control group.

\section{Cortical Thickness: Structural results}

Cortical thickness analyses revealed the presence of atrophy in the aMCI group relative to the control group in the bilateral premotor cortex, the medial temporal and occipito-temporoparietal regions (precuneus, angular and lingual gyrus, occipital gyrus, fusiform gyrus), the left ITG/MTG and ventrolateral/medial prefrontal cortex, as well as the right MTG and dorsolateral/medial prefrontal cortex (Figure 2b). Additional post-hoc whole brain regression analyses were conducted to explore the relation between six clusters of functional activity which are part of the semantic network and cortical thickness in the aMCI group. The clusters of peak MEG activity included the right ATL, left and right pMTG, two subregions of the right IPC, and the right fusiform gyrus. No significant negative correlation emerged between the extent of functional activation within any of these clusters and cortical thickness, showing that the pattern of functional hyperactivation found in the aMCI group in regions of the semantic network was not significantly associated with a decrease in cortical thickness (atrophy) in other brain regions. However, a significant positive correlation was found with the degree of activation in two regions

of the semantic network: right ATL/temporal pole activation in aMCI participants was positively 
correlated with cortical thickness in the right ITG/fusiform gyrus, and the right ITG/fusiform activation with the left pMTG/fusiform region and primary visual cortex (BA17).

\section{Discussion}

The main purpose of this study was to investigate the brain mechanisms associated with semantic processing of famous faces in aMCI individuals. A group of aMCI patients and a matched group of healthy seniors completed a computerized semantic categorization task while cortical activation was recorded using MEG. In line with previous studies, aMCI patients performed significantly worse than healthy controls on the semantic task, as evidenced by lower accuracy rates and longer response times. MEG results revealed a main pattern of hyperactivation in the aMCI group within several regions of the semantic network, along with a milder pattern of hypoactivation. This network included the ATL region, which has been previously associated with the storage of conceptual knowledge and processing of semantic information at an abstract and amodal level (Patterson et al., 2007; Ralph et al., 2017). It also included the IPC and pMTG, which have been associated with semantic executive control processes, including task-driven semantic processing and retrieval of relevant information in the conceptual store (Jefferies, 2013; Noonan et al., 2013; Ralph et al., 2017; Whitney et al., 2011). A similar phenomenon of hyperactivation has been previously observed in aMCI engaged in episodic memory tasks (Dickerson et al., 2005; 2008; Hämäläinen, Pihlajamäki, et al., 2007; Kircher et al., 2007). To our knowledge, however, this the first study to observe such a pattern in response to a semantic task explicitly probing famous person knowledge.

At the behavioural level, aMCI participants were slower and less accurate than age- and education-matched healthy controls on the famous faces semantic task. These results corroborate those of previous studies which found that aMCI patients performed worse on various tasks probing semantic knowledge about famous persons and suggests that this specific type of knowledge may be particularly prone to semantic breakdown early in the disease process (Barbeau et al., 2012; Brambati et al., 2012; Clague et al., 2011; Estevez-Gonzalez et al., 2004; Joubert et al., 2010; Joubert et al., 2008; Thompson et al., 2002; Vogel et al., 2005). Interestingly, our aMCI patients were not more impaired than controls on more basic semantic tests, such as the PPTT and the BNT. Performance on the famous faces test was not correlated either with performance on 
these standard neuropsychological tests tapping semantic knowledge. These results support the idea that tests tapping famous person knowledge may be particularly sensitive and useful in probing semantic deficits in prodromal $\mathrm{AD}$, when compared to more conventional neuropsychological measures of object knowledge.

Since a large proportion of aMCI patients are likely to develop AD over the following years, semantic deficits documented in aMCI may reflect the vulnerability of SM in the early stage of $\mathrm{AD}$. In fact, semantic deficits have been reported to reflect the earliest features of cognitive decline in the disease. Two retrospective epidemiological studies in large population cohorts showed that semantic deficits represented the earliest signs of cognitive decline in individuals who later developed AD (Wilson et al., 2011) and appeared as early as 12 years before diagnosis was established (Amieva et al., 2008). Clinically speaking, these semantic deficits may go more unnoticed than the day-to-day episodic memory impairment, although one study reported a robust relation between semantic disruption in aMCI patients and difficulties in everyday functioning (Kirchberg et al., 2012). Although the aMCI group was not as good the control group on the famous faces task, the aMCI group showed normal performance on visuoperceptual tests, including a facial perception test (Benton Facial Recognition Test) (see Table 1). This seems to suggest that the semantic deficits found on the famous faces test were not due to specific difficulties in face perception in the aMCI group.

At the structural level, the pattern of widespread atrophy found in the aMCI group corroborates results of previous studies investigating the localization cortical grey-matter (GM) loss in aMCI patients (Balthazar et al., 2009; Derflinger et al., 2011; Hämäläinen, Tervo, et al., 2007; Whitwell et al., 2007; Zheng et al., 2014). Atrophy within specific brain regions has also been associated with a higher risk of conversion to $\mathrm{AD}$ in aMCI patients. For instance, a reduction of hippocampal volume and cortical thickness in the medial and lateral temporal, parietal and frontal lobes was visible up to two years prior to clinical diagnosis of $\mathrm{AD}$, relative to nonconverters (deToledo-Morrell et al., 2004; Devanand et al., 2012; Risacher \& Saykin, 2013). Risacher et al. (2009) also found a similar pattern of cortical atrophy within the temporal region in patients who converted from MCI to $\mathrm{AD}$ within a year in comparison with non-converters. 
At the functional level, the goal of this study was to investigate the neural correlates of the semantic impairment in aMCI using MEG, a neuroimaging technique offering both excellent temporal and spatial resolution. More specifically, the aim was to determine if certain areas of a semantic network were functionally altered, beyond medial temporal lobe regions typically involved in the episodic memory impairment in aMCI. Source localization analyses performed on the MEG signal showed areas of both hyperactivation and hypoactivation in aMCI patients when compared to healthy older controls related to the semantic task.

Several regions of the semantic network were found to be hyperactivated in the aMCI group, the most important one being the right ATL, but also the right fusiform gyrus, prefrontal cortex, and bilateral pMTG. Regarding the specific role of the right ATL activation, neuroanatomical models of SM have proposed that the ventrolateral ATL acts as an associative region where concepts are represented and processed at an abstract and amodal level (Lambon Ralph, Pobric, \& Jefferies, 2009; Patterson et al., 2007; Ralph et al., 2017; Visser et al., 2010). The model, initially described by Patterson (2007) and recently updated by Ralph et al. (2017), was based partly on semantic dementia (SD), a neurodegenerative condition characterized by a progressive breakdown in conceptual knowledge, with atrophy initially predominating in the ATL region (typically in the left hemisphere and progressing bilaterally) (Brambati et al., 2009). In rarer cases of patients presenting with predominant right-sided ATL atrophy, progressive and predominant loss of semantic knowledge occurred mainly for famous and familiar persons (Busigny, Robaye, Dricot, \& Rossion, 2009; Gainotti, Barbier, \& Marra, 2003; Joubert et al., 2006). Thus, the right ATL region plays a critical role in the processing of person-based semantic knowledge across various modalities, including faces and voices (Gainotti et al., 2003; Joubert et al., 2006). As for the fusiform gyrus, which also showed hyperactivation in the aMCI group, it shares preferential connectivity with the visual systems, and responds more to visual/concrete concepts (Mion et al., 2010; Ralph et al., 2017). Although it is widely recognized for its role in face perception, it extends anteriorly in the temporal lobes and also plays a key role in personbased semantic processing (Brunyé, Moran, Holmes, Mahoney, \& Taylor, 2017; Haxby, Hoffman, \& Gobbini, 2002; Rangarajan et al., 2014).

Hyperactivation was also found in the right IPC (BA46/47) and bilateral pMTG in the aMCI group. The IPC and pMTG are considered, along with the ATL, to be key regions of the 
semantic network (Binder, Desai, Graves, \& Conant, 2009; Jefferies, 2013; Ralph et al., 2017). More specifically, they are assumed to play a predominant role in executive aspects of semantic processing, such as the selection, inhibition, manipulation and controlled retrieval of semantic information (Jefferies, 2013; Jefferies \& Lambon Ralph, 2006; Noonan et al., 2013; Ricci et al., 1999; Whitney et al., 2011). In fact, co-occurring activation of IPC and pMTG has been reported in several imaging studies during situations of high semantic control demands (Badre, Poldrack, Paré-Blagoev, Insler, \& Wagner, 2005; Bedny, McGill, \& Thompson-Schill, 2008; Noonan et al., 2013; Zempleni, Renken, Hoeks, Hoogduin, \& Stowe, 2007), highlighting the role of these regions in semantic controlled/executive processes. Typically, these semantic control regions are leftlateralized, but certain studies have shown bilateral activation of these regions during semantic processing (Donnelly, Allendorfer, \& Szaflarski, 2011; Noonan et al., 2013), and the role of their right hemisphere counterpart in semantic processing remains to be better understood, particularly in the context of visual or non-verbal semantic tasks.

In sum, specific patterns of functional hyperactivation were found in our aMCI group in regions thought to be involved both in both central aspects (the right ATL) and in executive aspects (IPC and pMTG) of semantic processing. Even though the task in the current study was not specifically designed to tap implicit vs. explicit semantic processes, MEG results nonetheless provide functional neuroimaging evidence in support of the idea that semantic difficulties in aMCI may reflect both difficulties in semantic controlled processes as well as a loss of conceptual knowledge at a more central level, as previously suggested (Brambati et al., 2012; Joubert et al., 2010; Tsukiura et al., 2010).

In order to better understand the patterns of functional activation that were found in the current study in aMCI patients, which may potentially reflect a range of different neural processes, from functional compensation to inefficiency in the context of pathology-related neural decline, additional post-hoc regression analyses were performed to clarify their role and their relationship with both task performance and cortical GM integrity. This was important in order to better understand the mechanisms underlying the hyperactivation found in regions of the semantic network in the aMCI group, and to establish whether it reflected attempted compensation for the degenerative processes associated with AD pathology. Although recent theories surrounding the notion of compensation have focused mostly on the mechanisms that promote performance in 
healthy older individuals (relative to younger ones), there is a growing body of evidence suggesting that compensation may also occur in the context of neurodegenerative diseases, particularly in their preclinical stages (Gregory et al., 2017; Scheller, Minkova, Leitner, \& Klöppel, 2014). These studies have contributed to further defining the notion of compensation and identifying relevant criteria allowing to differentiate hyperactivation reflecting compensatory processes from neuronal inefficiency. Typically, neural compensation in the context of aging refers to situations where a specific network is recruited increasingly as a task becomes more difficult (compensation-related utilization of neural circuit hypothesis, or CRUNCH) (Reuter-Lorenz \& Cappell, 2008). Alternately, when a primary task-related network becomes altered in the face of pathology, the recruitment of additional, compensatory networks is necessary in order to maintain or improve performance (e.g. the scaffolding theory of aging and cognition, or STAC) (Barulli \& Stern, 2013; Park \& Reuter-Lorenz, 2009).

Here, specific patterns of hyperactivation found in semantic regions in the aMCI group may indicate compensatory neural activity specifically related to performing this task. This is the case for the clusters of hyperactivation found in the right ATL/temporal pole (TP; BA38) and in the right ITG/fusiform gyrus (BA20/37). Hyperactivation of these regions was positively correlated with performance (or associated with "preserved" performance) in the aMCI group but not in the control group. Moreover, there was a significant positive correlation between the extent of activation in these two regions and cortical thickness in other brain areas. Specifically, right ATL/TP activation was positively and significantly correlated with cortical GM in the right ITG/fusiform gyrus, while activation in the right ITG/fusiform gyrus was positively correlated with the cortical thickness of both the left pMTG/fusiform region and the primary visual area (BA17). Taken together, results from our study suggest that greater activation in specific regions of the semantic network in aMCI is associated with better performance on the semantic task (similar to that of healthy matched controls).

Contrariwise, there were no significant negative correlations between the intensity of activation within any of the six preselected semantic network regions and cortical thickness in other areas, suggesting that that these functional alterations may precede significant atrophy of the extended semantic network in aMCI. Moreover, hyperactivation patterns unrelated to performance in the aMCI group were found in several brain regions such as the right precuneus/posterior 
cingulate gyrus, inferior parietal regions and occipital subregions. It is possible that subtle changes in neurotransmitter function, hypometabolism/atrophy, or resting-state functional alterations (e.g. reduced deactivation of the default-mode network) in these regions may account for the observed effects in our aMCI group (Anchisi et al., 2005; Leal \& Yassa, 2013; Threlkeld, Jicha, Smith, \& Gold, 2011). Such hyperactivation may also reflect nonspecific neuronal activity associated with reduced global cognitive efficiency in patients, or increased dedifferentiation in activation between regions. In sum, although it is difficult to circumscribe their exact function, the nonspecific patterns of activation observed in our study appear to be more akin to the pathological burden than to semantic network related processes.

Moreover, hypoactivation was found within many of the atrophied regions in aMCI, while they completed the semantic task. Even though the current paradigm did not provide more in-depth information about the specific mechanisms underlying this pattern of hypoactivation, several reports in the literature have suggested reduced metabolic functioning within these regions in the aMCI and probable AD, making it a plausible explanation of the effects observed in this study (Anchisi et al., 2005; Wu et al., 2010). In addition, within the semantic network, activation of the left ITG in the aMCI group was inversely correlated with accuracy on the semantic categorization task (while correlation with task performance was positive in controls), suggesting that its contribution may become much less reliable as atrophy progresses, and supporting the possibility that both the structural and functional integrity of this region is diminished in aMCI.

In sum, these patterns of cortical activation highlight the presence of functional alterations within the semantic network in aMCI patients. Among the regions concerned by these changes, hyperactivation of the right ATL might represent a specific compensatory mechanism that may allow aMCI patients to maintain a level of performance relatively similar to that of older controls. This phenomenon has been previously mostly observed in aMCI patients engaging in episodic memory tasks (Dickerson et al., 2005; Dickerson \& Sperling, 2008), but this is the first time that this effect has been reported during a semantic categorization task. Considering the pattern of hypoactivation and atrophy reported in the aMCI group in the left ATL region through cortical thickness analyses, it is also possible that this increased recruitment of right-sided ATL region of the semantic network occurring in the aMCI group constitutes a modestly successful attempt at upregulation of the network in order to compensate for structural and functional changes in 
contralateral ATL regions (Schapiro, McClelland, Welbourne, Rogers, \& Ralph, 2013). Overall, the patterns of MEG hyperactivation in aMCI patients may reflect the presence of physiological alterations in various regions of the semantic network, which precede the occurrence of structural abnormalities of the same network in prodromal AD. However, because hyperactivation of the right ATL region is specifically associated with a better performance in aMCI patients, it may reflect a compensatory mechanism similar to that documented by Dickerson and colleagues in the context of early episodic memory decline in MCI and AD (Dickerson et al., 2005; 2008).

Finally, the current study presents several limitations, which need to be mentioned. First of all, we did not have biomarkers as supporting evidence of AD (Albert et al., 2011), which may be useful in minimizing heterogeneity between MCI patients. Also, the sample size of both groups was somewhat small in the current study. However, this is due to the fact that it is very difficult to use MEG with older persons with cognitive decline. Indeed, the number of aMCI participants in the current study was narrowed down from a much larger pool of patients who could not be included primarily due to MEG compatibility but also to head and eye movement. Moreover, since the current study did not include longitudinal follow-up measurements or intra-subject comparisons on the variables of interest interpretations about the compensatory nature of the hyperactivation patterns documented in the aMCI group should remain cautious since some uncertainty persists regarding individual levels of performance, brain activity and structural load (Gregory et al., 2017). Similarly, although we attempted to control for several factors by matching our groups according to age and education levels, it would have been interesting to be able to correlate the results obtained with more explicit measures of cognitive and brain reserve, in order to be able to better account for the complexity of the patterns observed.

\section{Conclusion}

In conclusion, we studied semantic processing of famous faces in a group of aMCI patients and in a group of age and education-matched healthy older controls. aMCI patients presented with reduced accuracy rates and increased response times relative to controls on the semantic task. The aMCI group showed a relatively diffuse pattern of cortical atrophy. MEG-recorded functional activity revealed a complex pattern of hyperactivated and hypoactivated regions in the aMCI group relative to the control group. In the aMCI group, two clusters of hyperactivation in specific regions 
of the semantic network positively contributed to performance on the famous faces task, the right temporal pole and the ITG/fusiform region. Altogether, these results suggest that these patterns of task-related hyperactivation may serve as compensatory processes in the aMCI group, and that aMCI patients present with abnormal patterns of functional activation in regions extending beyond MTL structures. Overall, these results provide neurofunctional evidence that memory decline in aMCI is not limited to episodic memory, and that a distributed network of brain regions underlying SM may also be functionally compromised early in the disease process. With that being said, further studies addressing the patterns of functional connectivity and relationships with whitematter tract alterations would constitute a helpful complement in trying to better understand the dynamics and function of these patterns of hyperactivation. For instance, previous studies have revealed that low-performing older adults tend to show white-matter deficits in the medial temporal regions while simultaneously displaying greater success-related activity in the same region during an episodic memory task; a similar pattern is observable within the prefrontal region during executive tasks (Daselaar et al., 2013); therefore, it would be interesting to explore if comparable mechanisms are at work in the semantic network. Moreover, brain regions found to be hyperactivated in aMCI during the semantic categorization task showed overlap with those corresponding to the DMN. Because both SM decline and DMN alterations have been previously documented in MCI, an investigation of the relationship between these concurrent changes may be warranted in order to better understand how these complex dynamics already at work in the early stages of $\mathrm{AD}$ might help predict evolution to dementia in aMCI patients. 


\section{References}

Ad-Dab'bagh, Y., Singh, V., Robbins, S., Lerch, J., Lyttelton, O., Fombonne, E., \& Evans, A. C. (2005, Jun. 13-16). Native space cortical thickness measurement and the absence of correlation to cerebral volume. Paper presented at the Proceedings of the 11th Annual Meeting of the Organization for Human Brain Mapping, Toronto, CAN.

Adlam, A.-L. R., Bozeat, S., Arnold, R., Watson, P., \& Hodges, J. R. (2006). Semantic knowledge in mild cognitive impairment and mild Alzheimer's disease. Cortex, 42(5), 675-684.

Ahmed, S., Arnold, R., Thompson, S. A., Graham, K. S., \& Hodges, J. R. (2008). Naming of objects, faces and buildings in mild cognitive impairment. cortex, 44(6), 746-752.

Albert, M. S., DeKosky, S. T., Dickson, D., Dubois, B., Feldman, H. H., Fox, N. C., . . Petersen, R. C. (2011). The diagnosis of mild cognitive impairment due to Alzheimer's disease: Recommendations from the National Institute on Aging-Alzheimer's Association workgroups on diagnostic guidelines for Alzheimer's disease. Alzheimer's \& Dementia, 7(3), 270-279.

Ally, B. A. (2012). Using Pictures and Words To Understand Recognition Memory Deterioration in Amnestic Mild Cognitive Impairment and Alzheimer's Disease: A Review [Review]. Current neurology and neuroscience reports, 12(6), 687-694.

Amblard, C., Lapalme, E., \& Lina, J. M. (2004). Biomagnetic source detection by maximum entropy and graphical models. IEEE Transactions on Biomedical Engineering, 51(3), 427442.

Amieva, H., Le Goff, M., Millet, X., Orgogozo, J. M., Pérès, K., Barberger-Gateau, P., . . . Dartigues, J. F. (2008). Prodromal Alzheimer's disease: successive emergence of the clinical symptoms. Annals of neurology, 64(5), 492-498.

Anchisi, D., Borroni, B., Franceschi, M., Kerrouche, N., Kalbe, E., Beuthien-Beumann, B., . . . Marcone, A. (2005). Heterogeneity of brain glucose metabolism in mild cognitive impairment and clinical progression to Alzheimer disease. Archives of Neurology, 62(11), 1728-1733.

Badre, D., Poldrack, R. A., Paré-Blagoev, E. J., Insler, R. Z., \& Wagner, A. D. (2005). Dissociable controlled retrieval and generalized selection mechanisms in ventrolateral prefrontal cortex. Neuron, 47(6), 907-918. 
Balthazar, M., Yasuda, C. L., Lopes, T. M., Pereira, F. R. S., Damasceno, B. P., \& Cendes, F. (2011). Neural correlates of lexical-semantic memory. Dementia e Neuropsychologia, 5(2), 69-77.

Balthazar, M., Yasuda, C. L., Pereira, F. R., Pedro, T., Damasceno, B. P., \& Cendes, F. (2009). Differences in grey and white matter atrophy in amnestic mild cognitive impairment and mild Alzheimer's disease. European Journal of Neurology, 16(4), 468-474.

Barbeau, E. J., Didic, M., Joubert, S., Guedj, E., Koric, L., Felician, O., . . Ceccaldi, M. (2012). Extent and neural basis of semantic memory impairment in mild cognitive impairment. Journal of Alzheimer's Disease, 28(4), 823-837.

Barrett, S., \& Rugg, M. D. (1989). Event-related potentials and the semantic matching of faces. Neuropsychologia, 27(7), 913-922.

Barulli, D., \& Stern, Y. (2013). Efficiency, capacity, compensation, maintenance, plasticity: emerging concepts in cognitive reserve. Trends in cognitive sciences, 17(10), 502-509.

Bedny, M., McGill, M., \& Thompson-Schill, S. L. (2008). Semantic adaptation and competition during word comprehension. Cerebral Cortex, 18(11), 2574-2585.

Benton, A. L. (1994). Contributions to neuropsychological assessment: A clinical manual. New York: Oxford University Press.

Besson, M., Magne, C., \& Regnault, P. (2004). Le traitement du langage. L'imagerie fonctionnelle électrique (EEG) et magnétique (MEG): Ses applications en sciences cognitives, 185-216.

Binder, J. R., Desai, R. H., Graves, W. W., \& Conant, L. L. (2009). Where is the semantic system? A critical review and meta-analysis of 120 functional neuroimaging studies. Cerebral Cortex, 19(12), 2767-2796.

Brambati, S. M., Peters, F., Belleville, S., \& Joubert, S. (2012). Lack of semantic priming effects in famous person recognition in Mild Cognitive Impairment. cortex, 48(4), 414-420.

Brambati, S. M., Rankin, K. P., Narvid, J., Seeley, W. W., Dean, D., Rosen, H. J., . . GornoTempini, M. L. (2009). Atrophy progression in semantic dementia with asymmetric temporal involvement: a tensor-based morphometry study. Neurobiology of Aging, 30(1), 103-111. doi:S0197-4580(07)00218-7 [pii]

10.1016/j.neurobiolaging.2007.05.014 
Brunyé, T. T., Moran, J. M., Holmes, A., Mahoney, C. R., \& Taylor, H. A. (2017). Non-invasive brain stimulation targeting the right fusiform gyrus selectively increases working memory for faces. Brain and cognition, 113, 32-39.

Busigny, T., Robaye, L., Dricot, L., \& Rossion, B. (2009). Right anterior temporal lobe atrophy and person-based semantic defect: a detailed case study. Neurocase, 15(6), 485-508.

Calero, M. D., Arnedo, M. L., Navarro, E., Ruiz-Pedrosa, M., \& Carnero, C. (2002). Usefulness of a 15-item version of the Boston Naming Test in neuropsychological assessment of loweducational elders with dementia. The Journals of Gerontology Series B: Psychological Sciences and Social Sciences, 57(2), 187-191.

Cardebat, D., Doyon, B., Puel, M., Goulet, P., \& Joanette, Y. (1990). Evocation lexicale formelle et sémantique chez des sujets normaux. Performances et dynamiques de production en fonction du sexe, de l'âge et du niveau d'étude. Acta Neurologica Belgica, 90(4), 207-217.

Carter, S. F., Caine, D., Burns, A., Herholz, K., Ralph, L., \& Matthew, A. (2012). Staging of the cognitive decline in Alzheimer's disease: insights from a detailed neuropsychological investigation of mild cognitive impairment and mild Alzheimer's disease. International Journal of Geriatric Psychiatry, 27(4), 423-432.

Chung, M. K., \& Taylor, J. (2004, Apr. 15-18). Diffusion smoothing on brain surface via finite element method. Paper presented at the Proceedings of the IEEE International Symposium on Biomedical Imaging: Nano to Macro, Arlington, VA, USA.

Chung, M. K., Worsley, K. J., Nacewicz, B. M., Dalton, K. M., \& Davidson, R. J. (2010). General multivariate linear modeling of surface shapes using SurfStat. Neuroimage, 53(2), 491505. doi:10.1016/j.neuroimage.2010.06.032

Clague, F., Graham, K. S., Thompson, S. A., \& Hodges, J. R. (2011). Is knowledge of famous people compromised in mild cognitive impairment? Cognitive and Behavioral Neurology, 24(3), 134-144.

Collins, D. L., Neelin, P., Peters, T. M., \& Evans, A. C. (1994). Automatic 3D intersubject registration of MR volumetric data in standardized Talairach space. Journal of Computer Assisted Tomography, 18(2), 192-205.

Cox, R. W. (1996). AFNI: software for analysis and visualization of functional magnetic resonance neuroimages. Computers and Biomedical research, 29(3), 162-173. 
Daselaar, S. M., Iyengar, V., Davis, S. W., Eklund, K., Hayes, S. M., \& Cabeza, R. E. (2013). Less wiring, more firing: low-performing older adults compensate for impaired white matter with greater neural activity. Cerebral Cortex, 25(4), 983-990.

Derflinger, S., Sorg, C., Gaser, C., Myers, N., Arsic, M., Kurz, A., . . Mühlau, M. (2011). Greymatter atrophy in Alzheimer's disease is asymmetric but not lateralized. Journal of Alzheimer's Disease, 25(2), 347-357.

deToledo-Morrell, L., Stoub, T. R., Bulgakova, M., Wilson, R. S., Bennett, D. A., Leurgans, S., . . Turner, D. A. (2004). MRI-derived entorhinal volume is a good predictor of conversion from MCI to AD. Neurobiology of aging, 25(9), 1197-1203.

Devanand, D. P., Bansal, R., Liu, J., Hao, X., Pradhaban, G., \& Peterson, B. S. (2012). MRI hippocampal and entorhinal cortex mapping in predicting conversion to Alzheimer's disease. Neuroimage, 60(3), 1622-1629.

Devlin, J. T., Russell, R. P., Davis, M. H., Price, C. J., Wilson, J., Moss, H. E., . . Tyler, L. K. (2000). Susceptibility-induced loss of signal: comparing PET and fMRI on a semantic task. Neuroimage, 11(6), 589-600.

Dickerson, B. C., Salat, D. H., Greve, D. N., Chua, E. F., Rand-Giovannetti, E., Rentz, D. M., .. . Blacker, D. (2005). Increased hippocampal activation in mild cognitive impairment compared to normal aging and AD. Neurology, 65(3), 404-411.

Dickerson, B. C., \& Sperling, R. A. (2008). Functional abnormalities of the medial temporal lobe memory system in mild cognitive impairment and Alzheimer's disease: insights from functional MRI studies. Neuropsychologia, 46(6), 1624-1635.

Dietl, T., Trautner, P., Staedtgen, M., Vannuchi, M., Mecklinger, A., Grunwald, T., ... Kurthen, M. (2005). Processing of famous faces and medial temporal lobe event-related potentials: a depth electrode study. Neuroimage, 25(2), 401-407.

Donnelly, K. M., Allendorfer, J. B., \& Szaflarski, J. P. (2011). Right hemispheric participation in semantic decision improves performance. Brain Research, 1419, 105-116. doi:http://dx.doi.org/10.1016/j.brainres.2011.08.065

Dudas, R. B., Clague, F., Thompson, S. A., Graham, K. S., \& Hodges, J. R. (2005). Episodic and semantic memory in mild cognitive impairment. Neuropsychologia, 43(9), 1266-1276.

Duong, A., Whitehead, V., Hanratty, K., \& Chertkow, H. (2006). The nature of lexico-semantic processing deficits in mild cognitive impairment. Neuropsychologia, 44(10), 1928-1935. 
Estevez-Gonzalez, A., Garcia-Sanchez, C., Boltes, A., Otermin, P., Pascual-Sedano, B., Gironell, A., \& Kulisevsky, J. (2004). Semantic knowledge of famous people in mild cognitive impairment and progression to Alzheimer's disease. Dementia and Geriatric Cognitive Disorders, 17(3), 188-195.

Federmeier, K. D., \& Kutas, M. (2001). Meaning and modality: Influences of context, semantic memory organization, and perceptual predictability on picture processing. Journal of Experimental Psychology-Learning Memory and Cognition, 27(1), 202-224.

Federmeier, K. D., Van Petten, C., Schwartz, T. J., \& Kutas, M. (2003). Sounds, words, sentences: age-related changes across levels of language processing. Psychology and aging, 18(4), 858-872.

Folstein, M. F., Folstein, S. E., \& McHugh, P. R. (1975). "Mini-mental state”: a practical method for grading the cognitive state of patients for the clinician. Journal of Psychiatric Research, 12(3), 189-198.

Gainotti, G., Barbier, A., \& Marra, C. (2003). Slowly progressive defect in recognition of familiar people in a patient with right anterior temporal atrophy. Brain, 126(4), 792-803.

Gigi, A., Babai, R., Penker, A., Hendler, T., \& Korczyn, A. D. (2010). Prefrontal compensatory mechanism may enable normal semantic memory performance in mild cognitive impairment (MCI). Journal of Neuroimaging, 20(2), 163-168.

Grabner, G., Janke, A. L., Budge, M. M., Smith, D., Pruessner, J., \& Collins, D. L. (2006, Oct 16). Symmetric atlasing and model based segmentation: an application to the hippocampus in older adults. Paper presented at the 9th International Conference of Medical Image Computing and Computer-Assisted Intervention (MICCAI), Copenhagen, DK.

Gregory, S., Long, J. D., Klöppel, S., Razi, A., Scheller, E., Minkova, L., . . Leavitt, B. R. (2017). Operationalizing compensation over time in neurodegenerative disease. Brain, 140(4), 1158-1165.

Grova, C., Daunizeau, J., Lina, J. M., Bénar, C. G., Benali, H., \& Gotman, J. (2006). Evaluation of EEG localization methods using realistic simulations of interictal spikes. Neuroimage, 29(3), 734-753.

Haidar, H., \& Soul, J. S. (2006). Measurement of cortical thickness in 3D brain MRI data: validation of the Laplacian method. Journal of Neuroimaging, 16(2), 146-153. 
Hämäläinen, A., Pihlajamäki, M., Tanila, H., Hänninen, T., Niskanen, E., Tervo, S., . . Soininen, H. (2007). Increased fMRI responses during encoding in mild cognitive impairment. Neurobiology of Aging, 28(12), 1889-1903.

Hämäläinen, A., Tervo, S., Grau-Olivares, M., Niskanen, E., Pennanen, C., Huuskonen, J., . . . Vanhanen, M. (2007). Voxel-based morphometry to detect brain atrophy in progressive mild cognitive impairment. Neuroimage, 37(4), 1122-1131.

Harbin, T. J., Marsh, G. R., \& Harvey, M. T. (1984). Differences in the late components of the event-related potential due to age and to semantic and non-semantic tasks. Electroencephalography and Clinical Neurophysiology/Evoked Potentials Section, 59(6), 489-496.

Haxby, J. V., Hoffman, E. A., \& Gobbini, M. I. (2002). Human neural systems for face recognition and social communication. [Review]. Biol Psychiatry, 51(1), 59-67.

Howard, D., \& Patterson, K. E. (1992). The Pyramids and Palm Trees Test: A test of semantic access from words and pictures: Thames Valley Test Company.

Jefferies, E. (2013). The neural basis of semantic cognition: converging evidence from neuropsychology, neuroimaging and TMS. Cortex, 49(3), 611-625.

Jefferies, E., \& Lambon Ralph, M. A. (2006). Semantic impairment in stroke aphasia versus semantic dementia: a case-series comparison. Brain, 129(Pt 8), 2132-2147.

Jemel, B., George, N., Olivares, E., Fiori, N., \& Renault, B. (1999). Event-related potentials to structural familiar face incongruity processing. Psychophysiology, 36(4), 437-452.

Jones, S. E., Buchbinder, B. R., \& Aharon, I. (2000). Three-dimensional mapping of cortical thickness using Laplace's Equation. Human brain mapping, 11(1), 12-32.

Joubert, S., Brambati, S. M., Ansado, J., Barbeau, E. J., Felician, O., Didic, M., . . Kergoat, M. J. (2010). The cognitive and neural expression of semantic memory impairment in mild cognitive impairment and early Alzheimer's disease. Neuropsychologia, 48(4), 978-988.

Joubert, S., Felician, O., Barbeau, E., Ranjeva, J.-P., Christophe, M., Didic, M., . . Ceccaldi, M. (2006). The right temporal lobe variant of frontotemporal dementia. Journal of Neurology, 253(11), 1447-1458.

Joubert, S., Felician, O., Barbeau, E. J., Didic, M., Poncet, M., \& Ceccaldi, M. (2008). Patterns of semantic memory impairment in Mild Cognitive Impairment. Behavioural Neurology, 19(1-2), 35-40. 
Kim, J. S., Singh, V., Lee, J. K., Lerch, J., Ad-Dab'bagh, Y., MacDonald, D., . . Evans, A. C. (2005). Automated 3-D extraction and evaluation of the inner and outer cortical surfaces using a Laplacian map and partial volume effect classification. Neuroimage, 27(1), 210221.

Kirchberg, B. C., Cohen, J. R., Adelsky, M. B., Buthorn, J. J., Gomar, J. J., Gordon, M., .. . Davies, P. (2012). Semantic distance abnormalities in mild cognitive impairment: their nature and relationship to function. American Journal of Psychiatry, 169(12), 1275-1283.

Kircher, T. T., Weis, S., Freymann, K., Erb, M., Jessen, F., Grodd, W., . . Leube, D. T. (2007). Hippocampal activation in patients with mild cognitive impairment is necessary for successful memory encoding. Journal of Neurology, Neurosurgery \& Psychiatry, 78(8), 812-818.

Kutas, M., \& Federmeier, K. D. (2011). Thirty years and counting: finding meaning in the N400 component of the event-related brain potential (ERP). [Review]. Annual review of psychology, 62, 621-647.

Kutas, M., \& Hillyard, S. A. (1980). Reading senseless sentences: brain potentials reflect semantic incongruity. Science, 207(4427), 203-205.

Kutas, M., \& Iragui, V. (1998). The N400 in a semantic categorization task across 6 decades. Electroencephalography and Clinical Neurophysiology/Evoked Potentials Section, 108(5), 456-471.

Lambon Ralph, M. A., Pobric, G., \& Jefferies, E. (2009). Conceptual Knowledge Is Underpinned by the Temporal Pole Bilaterally: Convergent Evidence from rTMS. Cerebral Cortex, 19(4), 832-838.

Lau, E., Almeida, D., Hines, P. C., \& Poeppel, D. (2009). A lexical basis for N400 context effects: evidence from MEG. Brain and Language, 111(3), 161-172.

Lau, E., Phillips, C., \& Poeppel, D. (2008). A cortical network for semantics: (de)constructing the N400. [Review]. Nature Reviews Neuroscience, 9(12), 920-933.

Lawton, M. P., \& Brody, E. M. (1969). Assessment of older people: self-maintaining and instrumental activities of daily living. Gerontologist, 9(3), 179-186.

Leal, S. L., \& Yassa, M. A. (2013). Perturbations of neural circuitry in aging, mild cognitive impairment, and Alzheimer's disease. Ageing Research Reviews, 12(3), 823-831. doi:http://dx.doi.org/10.1016/j.arr.2013.01.006 
Lerch, J. P., \& Evans, A. C. (2005). Cortical thickness analysis examined through power analysis and a population simulation. Neuroimage, 24(1), 163-173.

Levy, D. A., Bayley, P. J., \& Squire, L. R. (2004). The anatomy of semantic knowledge: Medial vs. lateral temporal lobe. Proceedings of the National Academy of Sciences of the United States of America, 101(17), 6710-6715.

Libon, D. J., Rascovsky, K., Powers, J., Irwin, D. J., Boller, A., Weinberg, D., . . Grossman, M. (2013). Comparative semantic profiles in semantic dementia and Alzheimer's disease. Brain, 136(8), 2497-2509.

MacDonald, D., Kabani, N., Avis, D., \& Evans, A. C. (2000). Automated 3-D extraction of inner and outer surfaces of cerebral cortex from MRI. Neuroimage, 12(3), 340-356.

Mion, M., Patterson, K., Acosta-Cabronero, J., Pengas, G., Izquierdo-Garcia, D., Hong, Y. T., .. . Nestor, P. J. (2010). What the left and right anterior fusiform gyri tell us about semantic memory. Brain, 133(11), 3256-3268.

Noonan, K. A., Jefferies, E., Visser, M., \& Ralph, M. A. L. (2013). Going beyond Inferior Prefrontal Involvement in Semantic Control: Evidence for the Additional Contribution of Dorsal Angular Gyrus and Posterior Middle Temporal Cortex. Journal of Cognitive Neuroscience, 25(11), 1824-1850.

Park, D. C., Lautenschlager, G., Hedden, T., Davidson, N. S., Smith, A. D., \& Smith, P. K. (2002). Models of visuospatial and verbal memory across the adult life span. Psychology and aging, 17(2), 299-320.

Park, D. C., \& Reuter-Lorenz, P. (2009). The adaptive brain: aging and neurocognitive scaffolding. Annual review of psychology, 60, 173-196.

Patterson, K., Nestor, P. J., \& Rogers, T. T. (2007). Where do you know what you know? The representation of semantic knowledge in the human brain. [Review]. Nature Reviews Neuroscience, 8(12), 976-987.

Petersen, R. C., Doody, R., Kurz, A., Mohs, R. C., Morris, J. C., Rabins, P. V., . . Winblad, B. (2001). Current concepts in mild cognitive impairment. Archives of neurology, 58(12), 1985-1992.

Ralph, M. L., Jefferies, E., Patterson, K., \& Rogers, T. T. (2017). The neural and computational bases of semantic cognition. Nat. Rev. Neurosci., 18, $42-55$. 
Rangarajan, V., Hermes, D., Foster, B. L., Weiner, K. S., Jacques, C., Grill-Spector, K., \& Parvizi, J. (2014). Electrical stimulation of the left and right human fusiform gyrus causes different effects in conscious face perception. Journal of Neuroscience, 34(38), 12828-12836.

Regard, M. (1981). Stroop Test-Victoria Version. Victoria, BC: Neuropsychological Laboratory, University of Victoria.

Reitan, R. M. (1955). The relation of the trail making test to organic brain damage. Journal of Consulting Psychology, 19(5), 393-394.

Reuter-Lorenz, P. A., \& Cappell, K. A. (2008). Neurocognitive aging and the compensation hypothesis. Current directions in psychological science, 17(3), 177-182.

Rey, A., Wallon, P., \& Mesmin, C. (1960). Test de la figure complexe de Rey. Paris: Les Éditions du Centre de Psychologie Appliquée.

Ricci, P. T., Zelkowicz, B. J., Nebes, R. D., Meltzer, C. C., Mintun, M. A., \& Becker, J. T. (1999). Functional neuroanatomy of semantic memory: Recognition of semantic associations. Neuroimage, 9(1), 88-96.

Risacher, S. L., \& Saykin, A. J. (2013). Neuroimaging and other biomarkers for Alzheimer's disease: the changing landscape of early detection. [Review]. Annual Review of Clinical Psychology, 9, 621-648.

Risacher, S. L., Saykin, A. J., Wes, J. D., Shen, L., Firpi, H. A., \& McDonald, B. C. (2009). Baseline MRI predictors of conversion from MCI to probable AD in the ADNI cohort. Current Alzheimer Research, 6(4), 347-361.

Schapiro, A. C., McClelland, J. L., Welbourne, S. R., Rogers, T. T., \& Ralph, M. A. L. (2013). Why bilateral damage is worse than unilateral damage to the brain. Journal of Cognitive Neuroscience, 25(12), 2107-2123.

Scheller, E., Minkova, L., Leitner, M., \& Klöppel, S. (2014). Attempted and successful compensation in preclinical and early manifest neurodegeneration-a review of task fMRI studies. Frontiers in psychiatry, 5.

Sled, J. G., Zijdenbos, A. P., \& Evans, A. C. (1998). A nonparametric method for automatic correction of intensity nonuniformity in MRI data. IEEE transactions on medical imaging, $17(1), 87-97$.

Smith, M., \& Halgren, E. (1987). Event-related potentials elicited by familiar and unfamiliar faces. Electroencephalography and clinical neurophysiology. Supplement, 40, 422. 
Smith, S. M., Matthews, P. M., \& Jezzard, P. (2001). Functional MRI: an introduction to methods: Oxford University Press.

Sowell, E. R., Peterson, B. S., Kan, E., Woods, R. P., Yoshii, J., Bansal, R., . . Toga, A. W. (2007). Sex differences in cortical thickness mapped in 176 healthy individuals between 7 and 87 years of age. Cerebral Cortex, 17(7), 1550-1560.

Taler, V., Klepousniotou, E., \& Phillips, N. A. (2009). Comprehension of lexical ambiguity in healthy aging, mild cognitive impairment, and mild Alzheimer's disease. Neuropsychologia, 47(5), 1332-1343.

Thompson, S. A., Graham, K. S., Patterson, K., Sahakian, B. J., \& Hodges, J. R. (2002). Is knowledge of famous people disproportionately impaired with patients with early and questionable Alzheimer's disease? Neuropsychology, 16(3), 344.

Threlkeld, Z. D., Jicha, G. A., Smith, C. D., \& Gold, B. T. (2011). Task deactivation reductions and atrophy within parietal default mode regions are overlapping but only weakly correlated in mild cognitive impairment. Journal of Alzheimer's Disease, 27(2), 415-427.

Tohka, J., Zijdenbos, A., \& Evans, A. (2004). Fast and robust parameter estimation for statistical partial volume models in brain MRI. Neuroimage, 23(1), 84-97.

Tsukiura, T., Mano, Y., Sekiguchi, A., Yomogida, Y., Hoshi, K., Kambara, T., . . Kawashima, R. (2010). Dissociable roles of the anterior temporal regions in successful encoding of memory for person identity information. Journal of Cognitive Neuroscience, 22(10), 22262237.

Tulving, E. (1972). Episodic and Semantic Memory1. Organization of memory, 381-402.

Van Der Linden, M., Coyette, F., Poitrenaud, J., Kalafat, M., Calicis, F., Wyns, C., \& Adam, S. (2004). L'épreuve de rappel libre / rappel indicé à 16 items (RL/RI-16) L'évaluation des troubles de la mémoire: Présentation de quatre tests de mémoire épisodique (avec leur étalonnage) (pp. 25-47). Marseilles, FR: Solal.

Van Der Meulen, M., Lederrey, C., Rieger, S. W., Van Assche, M., Schwartz, S., Vuilleumier, P., \& Assal, F. (2012). Associative and Semantic Memory Deficits in Amnestic Mild Cognitive Impairment as Revealed by Functional Magnetic Resonance Imaging. Cognitive and Behavioral Neurology, 25(4), 195-215. 
Verma, M., \& Howard, R. J. (2012). Semantic memory and language dysfunction in early Alzheimer's disease: a review. International Journal of Geriatric Psychiatry, 27(12), 12091217.

Visser, M., Jefferies, E., \& Ralph, M. A. L. (2010). Semantic processing in the anterior temporal lobes: a meta-analysis of the functional neuroimaging literature. Journal of Cognitive Neuroscience, 22(6), 1083-1094.

Vogel, A., Gade, A., Stokholm, J., \& Waldemar, G. (2005). Semantic memory impairment in the earliest phases of Alzheimer's disease. Dementia and Geriatric Cognitive Disorders, 19(23), 75-81.

Warrington, E. K., \& James, M. (1991). [VOSP: the visual object and space perception battery, Thames Valley Test Company].

Wechsler, D. (1997). WMS-III: Wechsler memory scale administration and scoring manual: Psychological Corporation.

Whitney, C., Kirk, M., O'Sullivan, J., Ralph, M. A. L., \& Jefferies, E. (2011). The neural organization of semantic control: TMS evidence for a distributed network in left inferior frontal and posterior middle temporal gyrus. Cerebral Cortex, 21(5), 1066-1075.

Whitwell, J. L., Przybelski, S. A., Weigand, S. D., Knopman, D. S., Boeve, B. F., Petersen, R. C., \& Jack, C. R. (2007). 3D maps from multiple MRI illustrate changing atrophy patterns as subjects progress from mild cognitive impairment to Alzheimer's disease. Brain, 130(7), 1777-1786.

Wilson, R. S., Leurgans, S. E., Boyle, P. A., \& Bennett, D. A. (2011). Cognitive decline in prodromal Alzheimer disease and mild cognitive impairment. Archives of Neurology, $68(3), 351-356$.

Woodard, J. L., Seidenberg, M., Nielson, K. A., Antuono, P., Guidotti, L., Durgerian, S., .. . Butts, A. (2009). Semantic memory activation in amnestic mild cognitive impairment. Brain, 132(8), 2068-2078.

Worsley, K. J., Liao, C. H., Aston, J., Petre, V., Duncan, G. H., Morales, F., \& Evans, A. C. (2002). A general statistical analysis for fMRI data. Neuroimage, 15(1), 1-15.

Wu, X., Chen, K., Yao, L., Ayutyanont, N., Langbaum, J. B., Fleisher, A., . . Alexander, G. E. (2010). Assessing the reliability to detect cerebral hypometabolism in probable 
Alzheimer's disease and amnestic mild cognitive impairment. Journal of neuroscience methods, 192(2), 277-285.

Yesavage, J. A., Brink, T. L., Rose, T. L., Lum, O., Huang, V., Adey, M., \& Leirer, V. O. (1983). Development and validation of a geriatric depression screening scale: a preliminary report. Journal of Psychiatric Research, 17(1), 37-49.

Zempleni, M.-Z., Renken, R., Hoeks, J. C. J., Hoogduin, J. M., \& Stowe, L. A. (2007). Semantic ambiguity processing in sentence context: Evidence from event-related fMRI. Neuroimage, 34(3), 1270-1279.

Zheng, D., Sun, H., Dong, X., Liu, B., Xu, Y., Chen, S., . . . Wang, X. (2014). Executive dysfunction and gray matter atrophy in amnestic mild cognitive impairment. Neurobiology of aging, 35(3), 548-555.

Zijdenbos, A., Forghani, R., \& Evans, A. (1998, Oct. 11-13). Automatic quantification of MS lesions in 3D MRI brain data sets: validation of INSECT. Paper presented at the Proceedings of the First International Medical Image Computing and Computer-Assisted Intervention (MICCAI), Cambridge, MA, USA. 


\section{Appendix A}

Figure 3. Trial design in the magnetoencephalography (MEG) semantic task

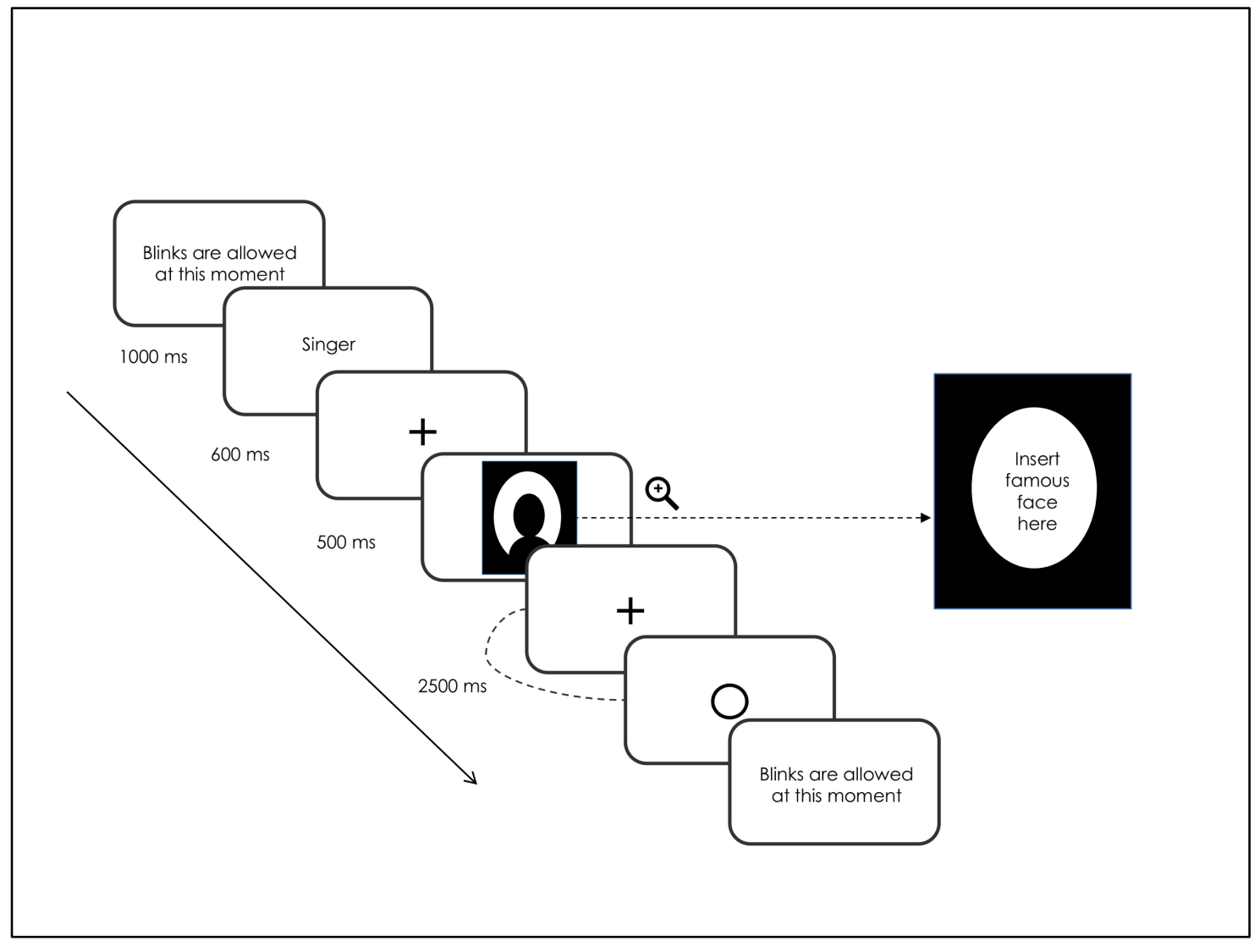




\section{Appendix B}

Table 3. List of the stimuli used in the semantic decision task

\begin{tabular}{llll}
\hline Actors & Singers & Athletes & Political figures \\
\hline Andrée Lachapelle & Barbara Streisand & Alexandre Despaties & André Boisclair \\
Béatrice Picard & Celine Dion & Ben Johnson & Bernard Landry \\
Brigitte Bardot & Charles Aznanour & Bruny Surin & Bill Clinton \\
Chantal Fontaine & Claude Dubois & Gilles Villeneuve & Diana Frances Spencer \\
Charles Lafortune & Dalida & Guy Carbonneau & Elizabeth A.M. Windsor \\
Gilles Latulippe & Dan Bigras & Guy Lafleur & Fidel Castro \\
Gaston Lepage & Daniel Bélanger & Jacques Demers & Georges W. Bush \\
Gérard Depardieu & Eric Lapointe & Jean Béliveau & Gérald Tremblay \\
Guylaine Tremblay & Félix Leclerc & Larry Robinson & Jacques Parizeau \\
Janine Sutto & Ginette Reno & Mario Lemieux & Jean Drapeau \\
Jean-François Pichette & Isabelle Boulay & Maurice Richard & Jimmy Carter \\
Juliette Huot & Jacques Brel & Michael Schumacher & Joe Clark \\
Karine Vanasse & John Lennon & Michel Bergeron & John F. Kennedy \\
Vivien Leigh & Johnny Cash & Mohammed Ali & Lucien Bouchard \\
Leonardo DiCaprio & Kevin Parent & Myriam Bédard & Margaret Thatcher \\
Marc Messier & Lara Fabian & Pat Burns & Pauline Marois \\
Paul Buissonneau & Laurence Jalbert & Patrick Roy & Pierre Elliot Trudeau \\
Pierre Lebeau & Luce Dufault & Scotty Bowman & René Lévesque \\
Rémi Girard & Michel Fugain & Roger Federer & Richard Nixon \\
Roger Moore & Michel Louvain & Saku Koivu & Robert Bourassa \\
Rose Ouellet & Michel Sardou & Serge Savard & Ronald Reagan \\
Jean-Paul Belmondo & Plume Latraverse & Sylvie Fréchette & Stephen Harper \\
Jean-Pierre Masson & Pierre Lalonde & Wayne Gretzky & Winston Churchill \\
Brigitte Paquette & Claude Léveillée & Yvan Cournoyer & Pierre Bourque \\
\hline & & & \\
\hline
\end{tabular}




\section{Appendix C}

Table 4. Correlations between brain activation and performance in the aMCI and controls

\begin{tabular}{lccccc}
\hline Region (Brodmann's Area) & $\mathbf{x}$ & $\mathbf{y}$ & $\mathbf{z}$ & Cluster Size & T value \\
\hline Brain activation positively correlated with performance in & aMCI group & \\
\hline Frontal regions & & & & & \\
R. Middle Frontal Gyrus (BA9) & 24 & 40 & 40 & 323 & 51.18 \\
L. Middle/Inferior Frontal Gyrus (BA44/45/46) & -38 & 18 & 22 & 371 & 46.94 \\
L. Medial Frontal Gyrus (BA11) & -8 & 64 & -18 & 81 & 5.74 \\
L. Middle Frontal Gyrus (BA6) & -40 & 4 & 54 & 57 & 6.99 \\
Temporal regions & & & & & \\
R. Parahippocampal Gyrus / Uncus (BA28) & 24 & -16 & -34 & 332 & 11.11 \\
R. Sup. Temporal Gyrus (BA39) & 62 & -60 & 30 & 115 & 4.55 \\
R. Superior Temporal Gyrus (BA38) & 38 & 16 & -24 & 56 & 15.59 \\
R. Inf. Temp. Gyrus / Fusiform (BA20/37) & 40 & -24 & -38 & 83 & 5.46 \\
L. Fusiform Gyrus (BA37) & -62 & -34 & -30 & 56 & 4.82 \\
Occipital regions & & & & & \\
R. Cuneus (BA17) & -10 & 32 & 28 & 171 & 25.36 \\
L. Inferior/Middle Occipital Gyrus (BA18/19) & -48 & -84 & -16 & 373 & 19.93 \\
Subcortical and limbic regions & & & & & \\
R. Insula (BA13) & 38 & 10 & 12 & 420 & 8.45 \\
R. Anterior Cingulate Gyrus (BA32) & -22 & 38 & 101 & 17.43 \\
L. Anterior Cingulate Gyrus (BA32) & & & & \\
\hline
\end{tabular}

Brain activation negatively correlated with performance in aMCI group

\section{Frontal regions}

R. Middle Frontal Gyrus (BA6)

$\begin{array}{rrrcr}20 & -12 & 68 & 145 & -23.72 \\ 24 & 18 & 60 & 121 & -10.78 \\ 50 & -0 & 48 & 147 & -12.12 \\ 10 & 6 & -14 & 57 & -4.79 \\ -32 & 16 & 52 & 1615 & -14.28\end{array}$

R. Superior Frontal Gyrus (BA6)

R. Precentral Gyrus

R. Subcallosal Gyrus (BA34)

\section{Temporal regions}

L. Middle Temporal Gyrus (BA20/21)

$\begin{array}{lllll}-58 & -38 & -16 & 203 & -19.75\end{array}$


L. Inferior Temporal Gyrus (BA20)

$$
\begin{array}{llll}
-56 & -16 & -38 & 105
\end{array}
$$

$-9.16$

L. Parahippocampal Gyrus (BA36)

$\begin{array}{lll}-28 & -36 & -8\end{array}$

81

$-10.25$

\section{Parietal regions}

L. Postcentral Gyrus (BA3)

$\begin{array}{lllll}-50 & -22 & 56 & 157 & -7.57\end{array}$

\section{Subcortical regions}

R. Thalamus

$\begin{array}{lllll}16 & -16 & 18 & 338 & -66.77\end{array}$

L. Lentiform Nucleus

$\begin{array}{lll}-24 & 4 & 14\end{array}$

124

$-51.30$

L. Thalamus

$\begin{array}{lll}-8 & -6 & -4\end{array}$

67

$-4.73$

\section{Brain activation positively correlated with performance in control group}

\section{Frontal regions}

R. Inferior Frontal Gyrus (BA9/45)

$\begin{array}{rrrrr}52 & 15 & 24 & 311 & 22.92 \\ 52 & 30 & 29 & 197 & 11.90 \\ 9 & 47 & 19 & 160 & 6.10 \\ -47 & 52 & -8 & 233 & 6.01 \\ -25 & -11 & 61 & 147 & 6.93\end{array}$

R. Middle Frontal Gyrus (BA46)

R. Medial Frontal Gyrus (BA10)

L. Middle Frontal Gyrus (BA10/47)

$-25-11-61$

6.93

\section{Temporal regions}

R. Middle Temporal Gyrus (BA21)

$\begin{array}{rrrrr}66 & -29 & -5 & 153 & 11.11 \\ 19 & -2 & -49 & 124 & 5.34 \\ -74 & -45 & 1 & 422 & 21.85 \\ -54 & -51 & -26 & 185 & 5.10\end{array}$

R. Uncus / Inf. Temporal Gyrus (BA36/20) 5.34

L. Middle Temporal Gyrus (BA21) 1.85

L. Fusiform Gyrus (BA37)

5.10

\section{Parietal regions}

R. Postcentral Gyrus

$\begin{array}{llll}64 & -14 & 26 & 223\end{array}$

4.91

R. Inf. Parietal /Supramarginal Gyrus (BA40)

$\begin{array}{llll}64 & -43 & 27 & 110\end{array}$

6.38

L. Inferior Parietal Lobule

$\begin{array}{llll}-69 & -64 & 41 & 172\end{array}$

7.23

L. Postcentral Gyrus

$\begin{array}{lll}-79 & -13 \quad 22\end{array}$

86

6.20

\section{Occipital regions}

R. Inferior Occipital Gyrus (BA18)

$\begin{array}{llll}47 & -88 & -16 & 221\end{array}$

6.65

R. Cuneus / Lingual Gyrus

$\begin{array}{llll}3 & -111 & -15 & 113\end{array}$

5.52

L. Middle Occipital Gyrus (BA18/19)

$\begin{array}{llll}-29 & -104 & 17 & 569\end{array}$

6.23

L. Lingual Gyrus

$\begin{array}{llll}-5 & -104 & -29 & 180\end{array}$

5.04

\section{Limbic regions}

R. Anterior Cingulate Gyrus (BA32)

$\begin{array}{llll}7 & 27 & 33 & 110\end{array}$

6.07 
